\title{
UN TENTATIVO DI ESPLORAZIONE DEI MOLTEPLICI NESSI DELLE NOZIONI ARISTOTELICHE DI GIUSTIZIA E INGIUSTIZIA, VIZIO E VIRTÙ, TRA PIANO ETICO E PIANO GIURIDICO
}

Arianna Fermani*

\begin{abstract}
RESUMO
Este artigo objetiva descobrir a multiplicidade de conexões entre justiça e virtude e entre injustiça e vício, bem como demonstrar como apenas o aceite da polivalência destas noções e a extrema adaptabilidade da relação entre elas permitem a co-existência nãocontraditória da diferença e mesmo afirmações contrárias no interior do texto de Aristóteles. Por exemplo, é apenas usando todos os sentidos da noção de justiça que podemos entender como a afirmação segunda a qual o mesmo ato injusto, sob uma certa perspectiva, é vício, enquanto sob outra não é vício, é possível. Um complexo cenário, sublinhado pelo Estagirita, extremamante rico de articulações internas (que devem ser respeitadas contra cada tentativa de redução), que se torna ainda mais complicado pelo contínuo jogo de transposições e de comparações entre ética e legitimidade legal estabelecidas pelo Filósofo.
\end{abstract}

PALAVRAS-CHAVE: Justiça. Virtude. Injustiça. Vício. Responsabilidade.

\begin{abstract}
This article aims to find out the multiplicity of connections between justice and virtue and between injustice and vice, and to

* Professora de Filosofia Antiga da Università degli Studi di Macerata - UNIMC/ Itália
\end{abstract}


show how only by the assumption of the polyvalence of these notions and of the extreme adaptability of the links between them allows the non-contradictory co-existence of different and even contrary affirmations inside the Aristotelian text. For instance, it's only by playing all the senses of the notion of justice, that we can understand how the contemporary affirmation that a same unjust act, under a certain point of view, is vicious, while, under another one, it isn't vicious, is possible. A complex scenery, the one outlined by the Stagirite, extremely rich of internal articulations (that demand to be respected against any attempt of reduction), that is further complicated by the continuous play of sliding and comparisons established by the Philosopher between ethical and legal level.

Key Words: Justice. Virtue. Injustice. Vice. Responsibility.

«Giustizia e ingiustizia si dicono in molti modi

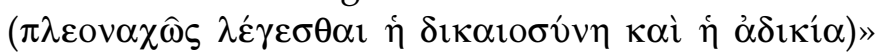
Aristotele, Etica Nicomachea, V, 21129 a 26-27

La notevole complessità del nesso tra giustizia e virtù che si viene ad instaurare all'interno della riflessione aristotelica costituisce da sempre un inesauribile terreno di riflessione ${ }^{2}$.

1 La traduzione dell'Etica Nicomachea, così come dei passi dell' Etica Eudemia e della Grande Etica che verranno esaminati in seguito, è di chi scrive. L'edizione critica di riferimento dell'Etica Nicomachea è quella curata da Franz Susemihl e rivista da Otto Apelt per la collana "Bibliotheca Teubneriana".

2 A questo proposito va anche detto che, come ha osservato H. J. Curzer, Aristotle's Account of the Virtue of Justice, "Apeiron», XXVIII, 3 (1995), pp. 207-238, p. 207, sebbene Aristotele dedichi un decimo dell'Etica Nicomachea alla questione della giustizia, e nonostante l'importanza che il tema della giustizia ricopre all'interno della trattazione delle virtù etiche, il tema è stato piuttosto sottovalutato dagli studiosi. Per un'accurata rassegna del dibattito filosofico sul tema della giustizia in e a partire da Aristotele, cfr. G. Zanetti, La nozione di

Educ. e Filos., Uberlândia, v. 21, n. 41, p. 169-211, jan./jun. 2007. 
L'intento di questo contributo, lungi dal voler dissodare un terreno così vasto e dal pretendere di cimentarsi con quella ricca serie di implicazioni (giuridiche, politiche, sociali ed economiche) e di risvolti che l'indagine aristotelica sulla giustizia chiama in causa ${ }^{3}$, è quello di limitarsi ad esaminare il rapporto tra giustizia e ingiustizia da un lato, e vizio e virtù dall'altro, mettendo a confronto, in relazione alle specifiche questioni in esame, piano etico e piano giuridico ${ }^{4}$. Un rapporto articolato, determinato

giustizia in Aristotele. Un percorso interpretativo, Il Mulino, Bologna 1993, pp. 913. Sul tema della giustizia e dei diritti in Aristotele, anche in rapporto alle teorie contemporanee dei diritti cfr. F. D. Miller, Jr., Aristotle and the Origins of Natural Rights, «The Review of Metaphysics» 49 (1996), pp. 873-907, ora in Lloyd P. Gerson (ed.), Aristotle Critical Assessments, 4 voll., vol. IV "Politics, Rhetoric and Aesthetics", Routledge, London-New York 1999, pp. 1-30; R. Kraut, Are there Natural Rights in Aristotle?, "The Review of Metaphysics» 49 (1996), pp. 755-774, ora in: Lloyd P. Gerson (ed.), Aristotle Critical Assessments..., cit., pp. 31-47; F. D. Miller Jr., Nature, Justice and Rights in Aristotle's Politics, Clarendon Press, Oxford 1995; W. von Leyden, Aristotle on Equality and Justice. His Political Argument, Macmillan, London 1985. Per un esame del tema della giustizia in rapporto alla questione dello stato e dei legami sociali, cfr. S. Stern-Gillet, Aristotles Philosophy of Friendship, State University of New York Press 1995, in particolare cap. 7: "Friendship, Justice, and the State", pp. 147-169.

3 Come ha infatti osservato Zanetti, Nozione di giustizia..., pp. 15-16: «Affrontare l'intera dottrina della giustizia di Aristotele è attualmente un'impresa tremenda, e ben difficilmente realizzabile senza ricorrere ad approssimazioni e superficialità anche gravi».

4 L'interesse di Aristotele per l'ambito giuridico, d'altra parte, non è certamente marginale: «Aristotele non si è occupato di diritto solo in modo tangenziale e nell'ottica di altre discipline... un po' come nel caso della matematica e della musica, ma ne ha ben colto la specificità ed ha investito e fatto investire notevolissime energie proprio nel sistematico studio dell'argomento» (L. Rossetti, Non solo dottrine politiche. L'apporto di Platone, Aristotele, Teofrasto e altri peripatetici alla cultura giuridica, in: M. Migliori (ed.), Gigantomachia. Convergenze e divergenze tra Platone e Aristotele, Morcelliana, Brescia 2002, p. 369). D'altra parte l'interesse aristotelico, e peripatetico in generale, per la legge e per l'esperienza giuridica è attestato dal fatto che, come ricorda E. Berti, Il filosofo e la città secondo Aristotele, in: Tatasciore C. (a cura di), I filosofi e la città, La Città del Sole, Napoli 2003, p. 49: «Aristotele... raccolse, probabilmente non da solo, ma con l'aiuto dei suoi amici e collaboratori, più di cento costituzioni, di cui si è rimasta soltanto la Costituzione degli Ateniesi. Sappiamo che fece anche raccolte di leggi e di proverbi». 
dall'applicazione costante di molteplici e irriducibili modelli esplicativi, che si complessifica ulteriormente nell' esplorazione, resa necessaria dallo stesso discorso aristotelico, di un altro fondamentale nesso: quello tra azioni giuste e azioni ingiuste da un lato, vizio e virtù dall'altro. Un nesso che, mentre a prima vista sembrerebbe reduplicare quello tra giustizia e virtù (e ingiustizia e vizio) o comunque muoversi sulla falsariga di esso, si rivela in realtà intrinsecamente connotato di sottili ma profonde dissomiglianze rispetto al rapporto precedentemente instaurato. Tale questione apre un ulteriore ventaglio di problemi da passare in rassegna e un groviglio, non sempre facilmente districabile, di nodi problematici. Nel tentativo di attraversamento di questo vero e proprio crocevia di questioni ci si atterrà principalmente al testo di Etica Nicomachea $\mathrm{V}^{5}$, che si cercherà di ripercorrere in maniera analitica in alcuni passaggi cruciali, allargando lo sguardo anche ad altri luoghi della stessa Etica Nicomachea, ad altri passi delle altre due Etiche aristoteliche e della Retorica.

\section{Giustizia e ingiustizia: vizio-virtù/tutto-parte}

In primo luogo si tratta di ricostruire l'intelaiatura generale della questione, così come viene fornita dallo Stagirita in Etica Nicomachea V 3 , in cui la giustizia è descritta come «virtù completa»

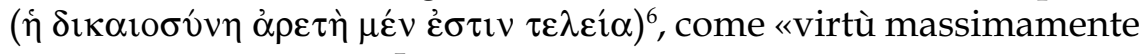
eccellente» $(\kappa \rho \alpha \tau i \sigma \tau \eta)^{7}$ e massimamente degna di ammirazione. La sua eccellenza, spiega Aristotele subito dopo, deriva dal fatto

\footnotetext{
5 «Aristotele si occupò ampiamente della giustizia... soprattutto nell'Etica, in cui vi dedicò tutto il libro $\mathrm{V}$, che costituì per tutto il Medioevo il fondamento del diritto naturale» (N. Quitidamo, Il concetto filosofico di "giustizia" in Aristotele e la sua inerenza col diritto, Stabilimento Tipografico Editoriale, Napoli 1935, p. $8)$.

6 Etica Nicomachea, V, 3, 1129 b 26.

7 Etica Nicomachea, V, 3, 1129 b 27: «la giustizia è ritenuta come la virtù più eccellente e... né la stella della sera né la stella del mattino sono altrettanto degne di ammirazione» (Aristotele, Etica Nicomachea, V, 3, 1129 b 27-29).
}

Educ. e Filos., Uberlândia, v. 21, n. 41, p. 169-211, jan./jun. 2007. 
che colui che la possiede è capace di esercitare la virtù verso il

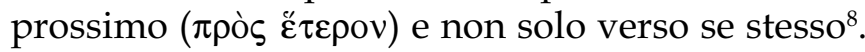

E se la giustizia intesa in questo senso, non è una parte della

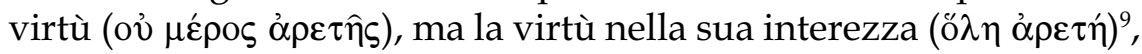
analogamente, l'ingiustizia non si configura solo come «una parte del vizio ( $\mu \varepsilon ́ \rho \circ \varsigma \kappa \alpha \kappa i \alpha \varsigma) »$, ma come «la totalità del vizio (ő $\lambda \eta$ $\left.\kappa \alpha \kappa^{\prime} \alpha\right) »^{10}$. In questo senso, come ha osservato giustamente Curzer ${ }^{11}$, la giustizia generale non è da intendere come una virtù accanto alle altre, quanto piuttosto come una virtù "di secondo ordine". La giustizia, quindi, è virtù, ed anzi la summa della virtù ${ }^{12}$ («nella giustizia è compresa ogni virtù» recita il proverbio $\left.{ }^{13}\right)$, e la sua peculiarità risiede, appunto, nel suo rivolgersi verso il prossimo

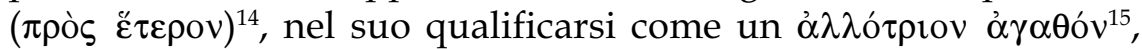
come un bene di altri.

Ma insieme ed oltre questa giustizia come ö $\lambda o v$, cioè come intero, c'è anche, come è noto, una virtù come parte, come $\mu$ ćpos della virtù totale. Il capitolo 4 del V libro dell'Etica Nicomachea inizia proprio con l'invito aristotelico ad esaminare

8 Etica Nicomachea, V, 3, 1129 b 32-33.

9 Etica Nicomachea, V, 3, 1130 a 9-10.

${ }^{10}$ Etica Nicomachea, V, 3, 1130 a 10-11.

${ }^{11}$ Curzer, Aristotle's Account..., p. 209.

${ }^{12}$ Sul tema del rapporto tra giustizia e virtù in Aristotele cfr. anche G. Santas, Goodness and Justice. Plato, Aristotle, and the Moderns, Blackwell, Oxford 2001, cap. 8 , in particolare pp. 278 ss., il quale, sulla base di una serie di argomentazioni non ripercorribili in questa sede, arriva alla conclusione estrema e difficilmente condivisibile, secondo cui «Aristotle does not have a virtue ethics for justice» (p. 287).

${ }^{13}$ Etica Nicomachea, V, 3, 1129 a 30.

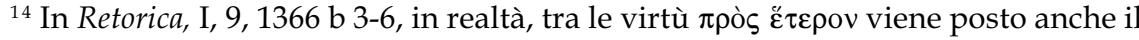
coraggio: «Necessariamente, se la virtù è una facoltà benefica, le maggiori virtù sono quelle che risultano più utili agli altri, e per questo si onorano soprattutto gli uomini dotati di giustizia e di coraggio» (Aristotele, Retorica, Introduzione di F. Montanari, Testo critico, traduzione e note a cura di M. Dorati, Mondadori, Milano 1996).

${ }^{15}$ Cfr. Etica Nicomachea, V, 10, 1134 b 5. 


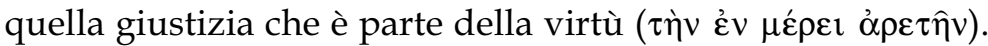
Infatti vi è una giustizia di questo tipo... e lo stesso vale anche per l'ingiustizia particolare ( $\left.\left.\alpha_{\delta} \delta \kappa^{\prime} \alpha \varsigma \tau \hat{\eta} \varsigma \kappa \alpha \tau \grave{\alpha} \mu \dot{\varepsilon} \rho\right)^{16}\right)^{16}$

e con la constatazione che

$c^{\prime}$ è un altro tipo di giustizia che deve essere intesa come

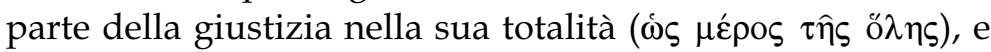

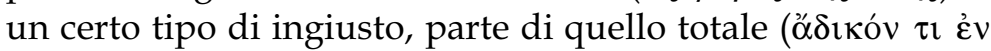

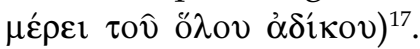

Quindi: c'è la giustizia come ó $\lambda$ ov, come virtù completa, che abbraccia e unifica una serie di virtù particolari ${ }^{18}$, tra cui la stessa giustizia particolare, e c'è l'ingiustizia come tutto, che si configura, a sua volta, come orizzonte di unificazione di una serie di vizi particolari, tra cui l'ingiustizia come $\mu$ épos.

Non a caso nella Retorica, dopo che la virtù è stata definita come «una facoltà in grado di procurare e preservare i beni, una facoltà che produce benefici grandi e numerosi, di ogni tipo e di ogni circostanza» ${ }^{19}$, si aggiunge che

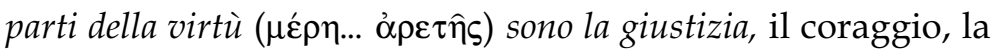
temperanza, la magnificenza, la magnanimità, la liberalità, $l^{\prime}$ assennatezza e la sapienza ${ }^{20}$ (corsivi miei).

E la giustizia, in quanto ö $\lambda o v$, e non in quanto $\pi \hat{\alpha} v^{21}$, non è un

\footnotetext{
${ }^{16}$ Etica Nicomachea, V, 4, 1130 a 14-16.

${ }^{17}$ Etica Nicomachea, V, 4, 1130 a 22-24.

${ }^{18}$ Si tratta di quelle virtù che sono state chiamate "di primo ordine": «general justice is not a virtue alongside the others... I shall indicate this difference by calling general justice a second order virtue while referring to courage, temperance, particular justice, etc. as first order virtue» (Curzer, Aristotle's Account..., p. 209).

${ }^{19}$ Retorica, I, 9, 1366 a 37-1366 b 1.

${ }^{20}$ Retorica, I, 9, 1366 b 1-3.

${ }^{21}$ Cfr. Metafisica, H, 6, 1045 a 9.
}

Educ. e Filos., Uberlândia, v. 21, n. 41, p. 169-211, jan./jun. 2007. 
semplice ammasso di parti, ma si configura piuttosto come

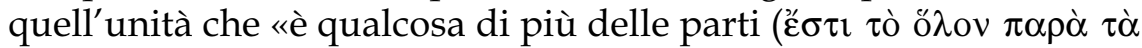
$\mu o ́ p ı \alpha) »^{22}$, come quella totalità organica che non si risolve nei suoi componenti, che trascende e, almeno formalmente, precede le parti di cui si compone. La logica che si viene a instaurare tra il tutto e le parti, inoltre, è illustrato dallo Stagirita attraverso il ricorso al nesso tra genere e specie:

cosicché è chiaro che vi è un'altra specie di ingiustizia, che è una parte della prima e ha lo stesso nome, dal momento che la definizione rientra nello stesso genere ${ }^{23}$.

L' "ingiustizia particolare" si configura, dunque, come una specie o un elemento del genere "ingiustizia totale". D'altra parte l'autorizzazione a ricondurre il nesso tutto-parti al rapporto tra genere e specie ci viene accordata dallo stesso Aristotele quando, in Metafisica $\Delta, 251023 \mathrm{~b}$ 17-19, passando in rassegna i vari significati della nozione di "parte", afferma che

si dicono parti anche quelle in cui la forma può essere divisa, a prescindere dalla quantità. Perciò si dice che le specie sono

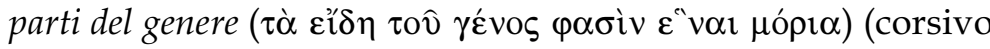
mio).

In questo senso la giustizia come intero, cioè come virtù totale, configurandosi come il genere a cui appartengono le specie-parti delle virtù particolari (e quindi anche della giustizia particolare), proprio in quanto genere deve rappresentare «il costitutivo primo delle definizioni, che è contenuto nell'essenza ${ }^{24}$, secondo la definizione di "genere" fornita nella Metafisica. Non è un caso,

${ }^{22}$ Metafisica, H, 6, 1145 a 10 (Aristotele, Metafisica, introduzione, traduzione, note e apparati di G. Reale, Appendice bibliografica di R. Radice, Bompiani, Milano 2000)

${ }^{23}$ Etica Nicomachea, V, 4, 1130 b 32-1130 b 1.

${ }^{24}$ Metafisica, $\Delta, 28,1024$ b 4-5.

Educ. e Filos., Uberlândia, v. 21, n. 41, p. 169-211, jan./jun. 2007. 
allora, che nel passo di Etica Nicomachea ricordato poco $\mathrm{fa}^{25}$, Aristotele faccia osservare che l'ingiustizia come parte ha lo stesso nome ${ }^{26}$ dell'ingiustizia come tutto, dal «momento che la definizione rientra nello stesso genere» e che, in quanto "tutto", è un «universale come abbracciante molte cose, in quanto si predica di ciascuna ( $\pi \mathrm{o} \lambda \lambda \dot{\alpha}$

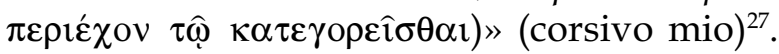

La giustizia come virtù completa, dunque, nel momento stesso in cui "abbraccia" le parti configurandosi come loro orizzonte di unificazione, imprime in esse il proprio indelebile sigillo. E se la giustizia intesa come tutto è la virtù, tutte le sue parti devono possedere la virtù come caratteristica essenziale ${ }^{28}$; analogamente,

${ }^{25}$ Cfr. Etica Nicomachea, V, 4, 1130 b 32-1130 b 1.

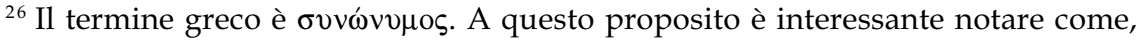
in Topici 123 a 28, Aristotele osservi che anche genere e specie sono sinonimi, confermando come il nesso tutto-parti possa essere letto in chiave "generespecie": "Sono infatti sinonimi il genere e la specie» (Aristotele, Topici, introduzione, traduzione e commento di A. Zadro, Loffredo, Napoli 1974).

${ }^{27}$ Metafisica D 26, 1023 b 30-31.

${ }^{28}$ In realtà in Etica Nicomachea, V, 3, 1130 a 11-14 si dice che virtù e giustizia, da un certo punto di vista sono la stessa cosa, mentre da un altro punto di vista non sono dotate della stessa essenza: «in che cosa, poi, differiscano la virtù e questo tipo di giustizia è chiaro da ciò che abbiamo detto: infatti, da un lato sono la stessa cosa, mentre dall'altro la loro essenza non è la stessa ma, in quanto si dirige verso il prossimo è giustizia, in quanto considerata, di per sé, come un determinato stato abituale è virtù (corsivo mio)». Natali (Aristotele, Etica Nicomachea, traduzione, introduzione e note di C. Natali, Laterza, Roma-Bari 1999, p. 488, n. 419) commenta: «quindi sono identiche solo da un certo punto di vista, come la salita, che coincide con la discesa anche se la loro definizione è diversa». A mio avviso il passo costituisce un ulteriore e cospicuo esempio della molteplicità di schemi adottati da Aristotele all'interno della propria riflessione. Se da un lato, infatti, come egli stesso afferma, giustizia e virtù sono la stessa cosa ( $\dot{\eta} \alpha \hat{\tau} \tau$ ஸ́), e sono la stessa cosa per tutta quella serie di nessi e di implicazioni già ricordati, dall'altro, cioè se la si considera nell'accezione di stato abituale, cioè come habitus, essa si configura come virtù. Ma Aristotele stesso, come vedremo, (cfr. § 4 "Giustizia e ingiustizia-azioni giuste e azioni ingiuste: la questione dell' habitus"), e a ulteriore dimostrazione della complessità del discorso, dedica proprio una parte della propria riflessione a dimostrare il nesso tra la giustizia e la virtù intesa come stato abituale. Sul rapporto di connessione e di distinzione tra la virtù e la giustizia cfr. anche A. Giuliani, La definizione aristotelica della giustizia. Metodo dialettico e analisi del linguaggio normativo, C. L. E. U. P., Perugia 1971, p. 80.

Educ. e Filos., Uberlândia, v. 21, n. 41, p. 169-211, jan./jun. 2007. 
se l'ingiustizia rappresenta la totalità del vizio, tutte le sue parti devono essere intrinsecamente ed essenzialmente connotate dal vizio. Come dire che non si può essere virtuosi senza essere, insieme, anche giusti, e che non si può essere viziosi senza essere, contemporaneamente, ingiusti ${ }^{29}$.

\section{Giustizia e ingiustizia: la virtù come giusto mezzo tra estremi} viziosi

$\mathrm{D}^{\prime}$ altra parte che la ingiustizia sia un vizio è confermato chiaramente della ripetuta e, insieme, problematica, definizione della giustizia come medietà, ovvero, al pari di tutte le altre virtù, come giusto mezzo tra due eccessi viziosi. Il quinto libro della Nicomachea, infatti, inizia proprio con questa precisa indicazione programmatica:

Dobbiamo ora occuparci della giustizia e dell'ingiustizia, di quali azioni le riguardino, quale medietà sia la giustizia e rispetto a che cosa il giusto costituisca l'elemento di medietà $»^{30}$.

Il fatto che la giustizia si configuri come giusto mezzo, poi, viene ribadito in Etica Nicomachea V 91133 b 32-33 in cui, all'affermazione che «la giustizia... è un certo tipo di medietà» si fa seguire una strana e inaspettata precisazione «ma non nello

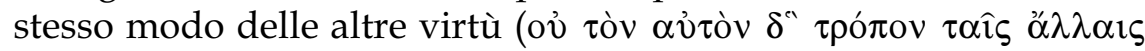
$\alpha \rho \varepsilon \tau \alpha \hat{\imath} \varsigma) »$. Se come tutte le altre virtù, dunque, la giustizia si configura come un giusto mezzo, d'altra parte questo giusto mezzo non è come gli altri e la giustizia si rivela una medietà del tutto sui generis. L'affermazione, che ha sollevato numerose perplessità e svariati tentativi di soluzione da parte degli studiosi, sembra essere supportata da quanto si legge nelle righe che seguono:

${ }^{29}$ Per un'analisi del nesso tra virtù e giustizia nel panorama complessivo della cultura greca cfr. A. W. H. Adkins, Merit and Responsibility. A Study in Greek Values, Clarendon Press, Oxford 1960, pp. 78 ss.

${ }^{30}$ Etica Nicomachea V, 1, 1129 a 3-5. 
E la giustizia è ciò per cui si dice che l'uomo giusto... è uno che non distribuisce $\mathrm{i}$ beni, a se stesso rispetto a un altro oppure a un altro rispetto a un terzo, in modo da avere lui stesso una parte maggiore di ciò che desidera e all'altro una parte minore... ma in modo da distribuire parti uguali secondo proporzione... Al contrario l'ingiustizia riguarda ciò che è ingiusto e questo è l'eccesso e il difetto ${ }^{31}$.

Quindi l'ingiustizia stessa, declinandosi sul duplice versante dell' eccesso e del difetto, del pretendere per sé più o meno di quanto si deve, cioè più o meno di quanto è giusto, veste i panni di entrambi gli estremi viziosi. La anomalia del giusto mezzo costituito dalla virtù della giustizia sembra allora essere risolta non tanto sulla base del fatto che la giustizia, come è stato osservato, non si oppone a due estremi viziosi ma solo ad uno ${ }^{32}$, cioè l'ingiustizia, ma in relazione alle caratteristiche della giustizia stessa in quanto medietà. Infatti, a differenza delle altre virtù, la giustizia è quella virtù attraverso la quale si realizza una adeguata distribuzione dei beni, quel giusto mezzo che permette di evitare gli eccessi opposti di

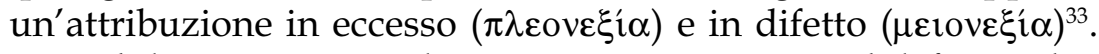

$\mathrm{D}^{\prime}$ altro canto, a ulteriore testimonianza del fatto che la molteplicità di schemi esplicativi e di modelli ermeneutici costituisce una costante del discorso aristotelico, in altri luoghi delle Etiche la

${ }^{31}$ Etica Nicomachea, V, 9, 1134 a 1-7. Analogamente, in Retorica I, 9, 1366 b 9-11 si afferma che «la giustizia è la virtù grazie alla quale ognuno possiede ciò che è suo ed è in accordo con legge; l'ingiustizia è ciò per cui si possiede quanto è di altri, non in conformità con la legge».

${ }^{32}$ Cfr. R. A. Gauthier - J. Y. Jolif, L'Éthique à Nicomaque, deuxième édition avec une introduction nouvelle, 4 voll., Éditions Peeters, Louvain-la Neuve, Paris 2002, p. 408.

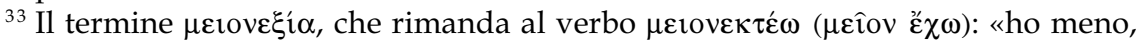
troppo poco, svantaggio, sono povero, sto in cattiva condizione, sto a corto di» (L. Rocci, Vocabolario greco-italiano, Società Editrice Dante Alighieri, Città di Castello 1985, p. 1196), in realtà, non compare nel corpus aristotelicum, ma viene efficacemente utilizzato da Curzer, Aristotle's Account..., pp. 220 ss. in

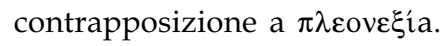

Educ. e Filos., Uberlândia, v. 21, n. 41, p. 169-211, jan./jun. 2007. 
giustizia viene a individuare una medietà, ma non rispetto agli estremi precedentemente individuati, quanto piuttosto rispetto al fare e al subire ingiustizia.

Di conseguenza è chiaro che la giustizia è una via di mezzo tra l'eccesso e il difetto e tra il molto e il poco. Infatti chi è ingiusto, per il fatto di compiere ingiustizia ha di più, mentre colui che subisce ingiustizia, per il fatto di subirla, ha di meno. Il giusto mezzo tra queste cose è il giusto (trad. mia),

si legge nella Grande Etica ${ }^{34}$.

Anche in questo contesto, comunque, l'ingiustizia è chiamata a svolgere il duplice ruolo di eccesso e difetto e la giustizia, come virtù, rappresenta la medietà tra questi eccessi. Una medietà che, come nel caso delle altre virtù etiche $\mathrm{e}$, forse, più di tutte le altre dal momento che deve anche fare i conti con la saggezza e l'imparzialità di chi giudica ${ }^{35}$, nonché con il vaglio accurato dei

${ }^{34}$ Aristotele, Grande Etica, I, 33, 1193 b 24-28. L'edizione critica di riferimento della Grande Etica è quella curata da Franz Susemihl per la collana “Biblioteca Teubneriana".

${ }^{35}$ Sulla figura del giudice cfr. Etica Nicomachea V 71132 a 20-24: «ricorrere al giudice è come ricorrere al giusto e il giudice, infatti, intende essere come la

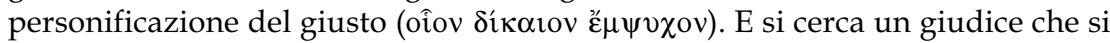
configuri come una figura imparziale, e alcuni chiamano i giudici "mediatori", intendendo dire che, se colgono il medio, coglieranno il giusto». Dalle fonti pervenuteci, però, sappiamo la realtà dei fatti era ben diversa. Nel mondo greco, come ci documentano M. Bretone, M. Talamanca, Il diritto in Grecia e a Roma, Laterza, Roma-Bari 1981, p. 67 «gli ateniesi sentivano come un rischio il processo, sia per quanto concerne le dikai private, sia, e ancor di più, per quanto riguarda le graphai e le altre accuse pubbliche». Infatti, «l'impossibilità di sottoporre ad un controllo e di valutare comparativamente le prove addotte, in senso contrastante, dalle parti, l'assenza di qualsiasi preparazione tecnicogiuridica dei giudici e dei logografi che eventualmente avessero preparato $i$ discorsi per i litiganti (per non parlare di questi ultimi) e l'impossibilità di approfondire l'interpretazione delle norme da applicare; la conseguente tendenza, chiaramente avvertibile presso i giurati, a fondare il proprio voto su una valutazione globale e soggettiva (e spesso arbitraria) della complessiva personalità delle parti e delle vicende tra di esse intercorse, al di là del punto 
vari elementi del caso, di tutte le opinioni e delle ragioni di una parte e dell'altra ${ }^{36}$, non ha niente a che vedere con la media matematica, valida e stabilita una volta per tutte, ma deve essere rintracciata volta per volta, calibrata attentamente in base alle circostanze, commisurata alla realtà fenomenologica, agli individui, alle situazioni a cui si trova di fronte caso per caso ${ }^{37}$. Aristotele, in questo senso, ha perfettamente compreso che, di fronte all'estrema mutevolezza e varietà delle situazioni, «l'applicazione meccanica delle regole potrebbe in certi casi portare a somma ingiustizia; ecco quindi la necessità di "distinguere" le singole situazioni: due situazioni possono essere in apparenza simili, prescindendo dal contesto delle circostanze. L'applicazione meccanica delle regole finirebbe così col determinare sostanzialmente un trattamento ineguale ${ }^{38}$. A differenza dei Pitagorici, che

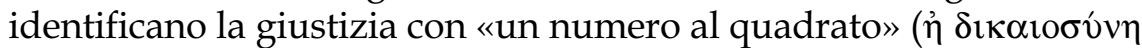

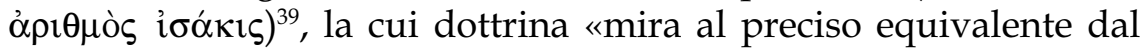
punto di vista quantitativo (contraccambio materiale, meccanico) $»^{40}$

di vista in discussione: tutto ciò giustificava senza ombra di dubbio una considerazione degli esiti del processo come un rischio» (Bretone-Talamanca, Diritto..., pp. 68-69). D'altra parte lo stesso Aristotele, in Etica Nicomachea V, 12, 1136 b 34-1137 a 4, avanza la possibilità che il giudice emani un verdetto errato: «Se invece ha giudicato ingiustamente pur nella piena cognizione di causa, il giudice stesso ci guadagna più del dovuto o in gratitudine o in vendetta. Cosicché, proprio come se avesse parte a un'azione ingiusta, anche chi giudica in modo ingiusto per i motivi appena ricordati si attribuisce più di quanto gli spetta. Infatti anche nel caso di colui che attribuì un terreno a quelle condizioni, non ci guadagnò un campo ma del denaro».

${ }^{36}$ L'argomentazione giuridica, al pari di quella dialettica, come ha osservato Giuliani, Definizione aristotelica..., p. 48, «deve procedere... caso per caso: non semplifica, ma distingue».

${ }^{37}$ Ecco perché, come è stato giustamente osservato, «non c'è mai la possibilità, nella condizione di complessità e imprevedibilità tipica dell'esperienza vitale, di dare una regola che non vada sottoposta ad una attenta valutazione rispetto alle condizioni concrete date» (M. Migliori, Misure ed etica tra quantità e qualità, «Tutto misure», 4, 1999, p. 29).

${ }^{38}$ Giuliani, Definizione aristotelica..., p. 92.

${ }^{39}$ Grande Etica I, 1, 1182 a 14.

${ }^{40}$ Giuliani, Definizione aristotelica..., p. 83.

Educ. e Filos., Uberlândia, v. 21, n. 41, p. 169-211, jan./jun. 2007. 
e da cui «i problemi della giustizia sono considerati semplici, misurabili, quantificabili» ${ }^{41}$, la nozione aristotelica di giustizia come giusto mezzo non ha nulla a che vedere con una misurazione di tipo matematico-quantitativo. D'altra parte, affermare che l'agire umano nel suo complesso non può essere misurato quantitativamente, che non può essere determinato numericamente e in modo assoluto, non significa affatto che deve essere condannato al soggettivismo e all'arbitrio. Oltre a una misura oggettiva, infatti, insiste Aristotele, esiste una misura "soggettiva", esiste un altro criterio: quello dell' uomo moralmente retto, dello $\sigma \pi \mathrm{ov \delta \alpha \hat { \imath } o s , ~ d i ~ c o l u i ~}$ che, essendo in perfette condizioni psicofisiche può fungere da regola.

Inoltre, come la particolarità della medietà virtuosa rappresentata dalla giustizia non inficia minimamente la sua natura di virtù, così l'ingiustizia, offrendosi come eccesso e come difetto, risulta doppiamente segnata dal vizio. Il legame strettissimo e indissolubile che si instaura tra virtù e giustizia, diventa dunque, nel rapporto tra ingiustizia e vizio, un legame a doppio nodo. Non stupisce affatto, allora, che in Etica Nicomachea, V, 4, 1130 a 28-29 si legga a chiare lettere che

riguardo a tutte le altre azioni ingiuste è sempre possibile ricondurle a una qualche forma di vizio $\left(\pi \varepsilon \rho \grave{\mu} \mu\right.$ " $v \tau \grave{\alpha} \alpha^{\prime} \lambda \lambda \alpha$

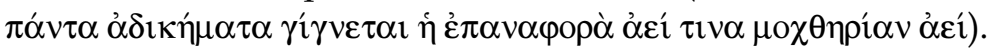

\section{Azioni giuste senza virtù-azioni ingiuste senza vizio}

Il percorso fin qui effettuato sembra attestare, all'interno della riflessione dello Stagirita, la presenza di un duplice binomio: giustizia e virtù da un lato, ingiustizia e vizio dall'altro.

Eppure ci sono alcune affermazioni aristoteliche che sembrano procedere in una direzione diametralmente opposta, escludendo la dimensione della virtù e quella del vizio, rispettivamente, dall'orizzonte della giustizia e dell'ingiustizia. Quest' esclusione,

\footnotetext{
${ }^{41}$ Giuliani, Definizione aristotelica..., p. 83.
} 
d'altra parte, risulta tanto spiazzante quanto denunciata esplicitamente e a più riprese da Aristotele stesso e affermata in modo assolutamente a-problematico. In quella interessante e acuta disamina, offerta da Etica Nicomachea,V, 10, circa le caratteristiche che un'azione deve possedere per essere definita ingiusta, Aristotele afferma in maniera esplicita che si possono compiere azioni che sono, contemporaneamente, ingiuste ma non viziose.

Infatti coloro che causano danni e commettono errori, agiscono sì ingiustamente, e le loro azioni sono sì ingiuste,

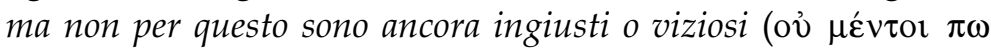

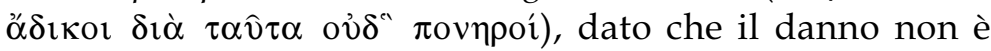

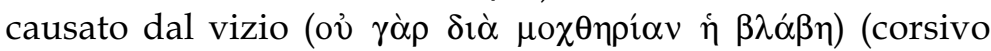
mio $^{42}$ (Etica Nicomachea, V, 10, 1135 b 22-25).

La complessità e, insieme, la centralità della questione, richiede un necessario allargamento del discorso a tutta quella parte della riflessione aristotelica ${ }^{43}$ che fa da cornice all' affermazione appena ricordata. Cercando di seguire passo per passo l'andamento del discorso aristotelico, si potrebbe schematizzare ed esemplificare i punti salienti dell'argomentazione nel modo che segue:

Si definiscono azioni ingiuste quelle azioni compiute:

a) consapevolmente ( $(\dot{i} \delta \dot{\omega} \varsigma)$

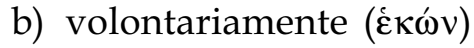

Tra le azioni compiute volontariamente, però,

(b1) alcune sono compiute con deliberazione e con scelta

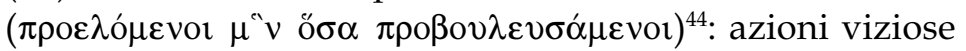
(b2) altre sono compite senza deliberazione e senza scelta,

${ }^{42}$ Etica Nicomachea, V, 10, 1135 b 22-24.

${ }^{43}$ Cfr., in particolare, Etica Nicomachea, V, 10, 1135 a 9 ss.

${ }^{44}$ Etica Nicomachea, V, 10, 1135 b 10.

Educ. e Filos., Uberlândia, v. 21, n. 41, p. 169-211, jan./jun. 2007. 


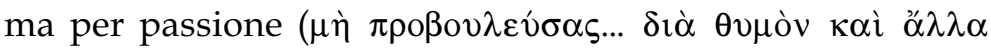
$\pi \alpha \dot{\theta} \theta \eta)^{45}$ : azioni non viziose.

L'argomentazione aristotelica, profondamente innervata di sottili e problematici distinguo, invita dunque a procedere con cautela46 e ad esaminare l'argomentazione punto per punto per provare a risolvere la problematicità del nesso giustizia-virtù / ingiustizia-vizio evidenziato poco fa.

\section{a) Ignoranza e costrizione-consapevolezza e volontarietà: tra imputabilità e non imputabilità dell'azione ingiusta}

Le prime due caratteristiche delineate, cioè la consapevolezza e la volontarietà, rappresentano gli elementi costitutivi dell'azione giusta e di quella ingiusta, nonché un fondamentale elemento discriminante rispetto alle altre azioni ${ }^{47}$. Se questo è vero, e contrario, l'ignoranza e la costrizione devono necessariamente costituire le due cause fondamentali della non imputabilità di un'azione ingiusta ${ }^{48}$.

${ }^{45}$ Etica Nicomachea, V, 10, 1135 b 20-21.

${ }^{46}$ Molto pertinente, in questo senso, l'osservazione di M. D'Avenia, L'aporia del bene apparente. Le dimensioni cognitive delle virtù morali in Aristotele, Vita e Pensiero, Milano 1998, pp. 245-246, secondo cui, «con il termine 'volontario', Aristotele intende non tanto 'ciò che si compie volontariamente' (che richiede la deliberazione...) quanto piuttosto 'ciò che procede in modo spontaneo dalla natura di qualcosa'. In questo senso, il significato che ha oggi per noi il termine 'volontario' (e che corrisponde alla proa...resij aristotelica) può risultare

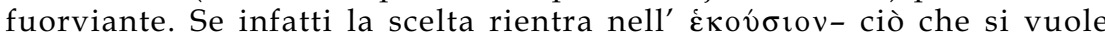
deliberatamente lo si vuole perché è comunque termine proprio di un principio che costituisce la natura umana-, non vale il contrario- non tutto ciò che

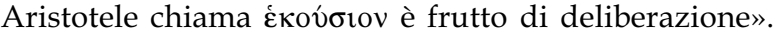

${ }^{47}$ Come ha osservato Giuliani, Definizione aristotelica..., p. 87: «guardare alla volontarietà dell'atto significa guardare alla imputabilità del soggetto».

${ }^{48}$ Sembra interessante notare come il diritto contemporaneo ponga esattamente le stesse condizioni di non punibilità di un reato poste da Aristotele: recitano infatti gli artt. 45 e 46 del codice penale che «non è punibile chi ha commesso il fatto per caso fortuito o per forza maggiore» e che "non è punibile chi ha commesso il fatto per esservi stato da altri costretto, mediante violenza fisica alla quale non poteva resistere o comunque sottrarsi». 
Si tratta due elementi che, nella riflessione dello Stagirita, risultano strettamente intrecciati:

quando l'ignoranza è la causa del compiere qualcosa, non si fa ciò volontariamente, cosicché non si commette ingiustizia,

si legge in Grande Etica, I, 33, 1195 a 25-26. Chi commette un'azione ingiusta a causa dell'ignoranza, cioè «non conoscendo né chi si danneggia, né il mezzo, né il fine» (Grande Etica, I, 33, 1195 a 24-25) dunque, non può essere detto ingiusto, ma semmai...

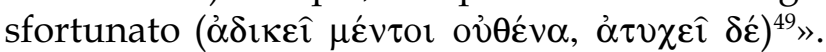

D'altro canto, però, anche a proposito dell'ignoranza Aristotele ritiene necessario introdurre una ulteriore e fondamentale distinzione:

quando invece la causa dell'ignoranza è lui stesso ed egli compie qualcosa a causa dell'ignoranza di cui è egli stesso causa, costui è colpevole d'ingiustizia e a buon diritto sarà chiamato ingiusto ${ }^{50}$.

Non sempre, quindi, l'ignoranza può essere addotta a sostegno della propria innocenza. L'esempio che viene fatto a questo proposito è quello dell'ubriachezza. Dice infatti Aristotele, introducendo, peraltro, una clausola di capitale importanza per la valutazione etica e giuridica di un reato e tuttora vigente nel diritto penale contemporaneo ${ }^{51}$, che

${ }^{49}$ Grande Etica, I, 33, 1095 a 24.

${ }^{50}$ Grande Etica, I, 33, 1095 a 28-31.

${ }^{51}$ Il nostro codice penale distingue, a questo proposito, una "ubriachezza derivante da caso fortuito o da forza maggiore" (art. 91), in base al quale "non è imputabile chi, nel momento in cui ha commesso il fatto, a causa di piena ubriachezza derivata da caso fortuito o da forza maggiore», e una "ubriachezza volontaria o colposa ovvero preordinata" (art. 92), in base al quale «l'ubriachezza non derivata da caso fortuito o da forza maggiore non esclude né diminuisce l'imputabilità» (C. E. Paliero, Codice Penale e Normativa Complementare, 2001-2002, quinta edizione, Raffaello Cortina Editore, Milano

Educ. e Filos., Uberlândia, v. 21, n. 41, p. 169-211, jan./jun. 2007. 
coloro che, ubriacandosi, compiono anche qualche azione malvagia, commettono ingiustizia. Infatti dell' ignoranza sono

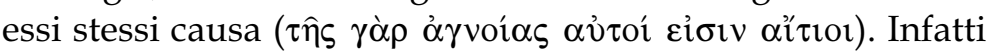
avevano la possibilità di non bere e di accorgersi così che stavano picchiando il proprio padre. E lo stesso vale anche per le altre forme di ignoranza che sorgono per colpa propria e in cui quelli che compiono ingiustizia conformemente ad esse sono ingiusti, mentre nelle cose delle quali essi stessi

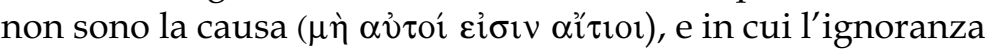
è la causa per cui essi hanno fatto ciò che hanno fatto, non sono ingiusti ${ }^{52}$.

Altro, dunque, è agire ingiustamente a causa dell'ignoranza, altro ancora è essere causa di quella stessa ignoranza e rendersi con ciò responsabili dell'azione ingiusta. Ecco quindi perché il filosofo afferma, aggiungendo peraltro una ulteriore interessante precisazione in merito alla naturalità e all'umanità di alcune passioni, che le azioni del primo tipo cioè, precisa, «quegli errori che si compiono, non solo in stato di ignoranza, ma proprio a causa

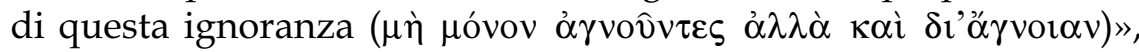
sono da considerarsi perdonabili ( $\sigma v \gamma \gamma \nu \omega \mu$ ovıкó), mentre quelli che sono compiuti «in uno stato di ignoranza, ma in uno stato di ignoranza non dovuto a una passione che non sia né naturale né

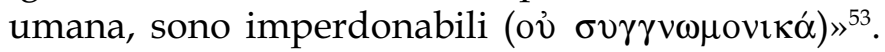

Ma tra i fattori di non imputabilità, come si è visto, oltre l'ignoranza c'è la costrizione: infatti, come non può essere considerata ingiusta in senso proprio l'azione di colui che uccida un individuo ignorando che si tratti di suo padre ${ }^{54}$, allo stesso modo

2001). Un ringraziamento particolare va rivolto, a questo proposito, a Patrizio Ciabocco, che attraverso le sue preziose indicazioni e i suoi fondamentali suggerimenti, mi ha fornito le coordinate per attraversare l'impervio terreno del diritto penale, rendendo possibile l'individuazione di alcuni significativi nessi tra diritto antico e contemporaneo.

${ }^{52}$ Grande Etica, I, 33, 1195 a 31-37.

${ }^{53}$ Etica Nicomachea, V, 10, 1136 a 6-9.

${ }^{54}$ Cfr. Etica Nicomachea, V, 10, 1135 a 28 ss. 
non può esserlo colui che viene costretto da qualcuno a picchiare qualcun altro ${ }^{55}$, osserva Aristotele.

Alla delimitazione in negativo dell'ambito dell'ingiustizia, segue dunque, come si è visto, l'individuazione delle condizioni di possibilità dell'attuazione dell'azione ingiusta stessa:

si commetterà ingiustizia e si agirà ingiustamente quando lo si farà volontariamente; al contrario, chi agisce sotto costrizione non può agire né in modo giusto né in modo ingiusto, se non per accidente: infatti compie azioni che per caso si rivelano essere giuste o ingiuste. Ma che un atto venga definito ingiusto dipende dal fatto che sia volontario e involontario ${ }^{56}$.

Detto in altri termini: si compie un'azione ingiusta perché la si vuole compiere (cfr. Etica Eudemia, II, 7, 1223 a 39: «il commettere

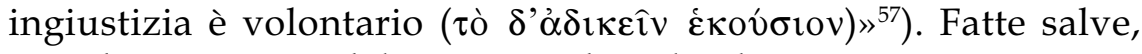
quindi, situazioni del tutto accidentali, che per caso vengono a rivelarsi sbagliate, come ad esempio quando si commette un errore o quando capita una disgrazia ${ }^{58}$, chi agisce ingiustamente lo deve fare in piena consapevolezza ${ }^{59}$, cioè «non ignorando né la persona,

55 «Come nell'ipotesi che uno, presa la mano di un altro, picchi un terzo, e che di certo non lo fa volontariamente; infatti l'azione non dipende da lui» (Etica Nicomachea, V, 10, 1134 b 27-28).

${ }^{56}$ Etica Nicomachea, V, 10, 1135 a 16-21.

${ }^{57}$ L'edizione critica di riferimento dell' Etica Eudemia è quella curata da R. R. Walzer e J. Mingay per la collana "Scriptorum classicorum bibliotheca oxoniensis".

${ }^{58} \mathrm{Di}$ grande interesse la fine distinzione attuata da Aristotele tra errore, inteso come ciò che viene inflitto in stato di ignoranza, e disgrazia, inteso come ciò che capita contro ogni aspettativa. Cfr., a questo proposito, Etica Nicomachea, V, 10, 1135 b 11 ss.; Retorica, I, 13, 1374 b 5-10: «è equo... non considerare alla pari gli errori e le azioni ingiuste, oppure gli errori e le sventure: le sventure sono impreviste e non derivano da malvagità, gli errori non sono imprevisti, ma non provengono da perversità, mentre le azioni ingiuste non sono impreviste e sono originate da perversità».

${ }^{59}$ In Retorica, I, 10, 1368 b 10-11 si legge: «si compie un'azione volontaria quando si è consapevoli».

Educ. e Filos., Uberlândia, v. 21, n. 41, p. 169-211, jan./jun. 2007. 
né il mezzo, né lo scopo dell'azione, come per esempio chi egli colpisce, con che cosa e a che scopo ${ }^{60}$ e volontariamente. Volontariamente, sì, ma non sempre in base ad una scelta e a una deliberazione, aggiunge lo Stagirita, introducendo una sottile ma fondamentale distinzione per l'intera riflessione etica e giuridica. Infatti è esattamente a questo livello che si situa il discrimen tra azioni semplicemente ingiuste e azioni ingiuste e, insieme, viziose.

Tra le azioni volontarie, alcune sono compiute per scelta, altre senza che siano state scelte e mentre scegliamo le cose su cui prima abbiamo deliberato, non scegliamo ciò su cui non abbiamo precedentemente deliberato $\left(\tau \hat{\omega} v \delta^{\prime \prime} \dot{\varepsilon} \kappa o v \sigma i \omega v\right.$

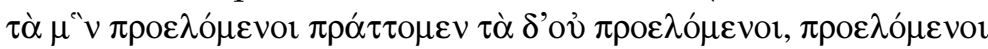

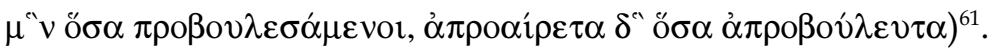

Se, quindi, si può dire che qualcuno commetta ingiustizia solo quando lo fa consapevolmente e volontariamente, d'altro canto, se questa azione non viene deliberata e successivamente scelta, non può essere considerata viziosa. Su questo aspetto la posizione di Aristotele non lascia adito al minimo dubbio:

non ogni atto volontario di ingiustizia comporta la presenza

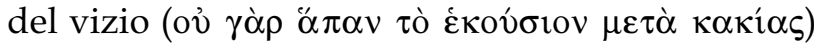

troviamo scritto in Etica Nicomachea, V, 15, 1138 a 34-35.

La contraddizione con quanto è stato rilevato precedentemente è palese e addirittura stridente. Come è possibile, dopo aver imperniato la giustizia e l'ingiustizia, rispettivamente sulla virtù e sul vizio, dopo aver esplicitamente instaurato una relazione non solo di somiglianza ma addirittura di identità ${ }^{62}$ tra questi elementi,

${ }^{60}$ Etica Nicomachea, V, 101135 a 25-26.

${ }^{61}$ Etica Nicomachea, V, 10, 1135 b 9-11.

${ }^{62}$ Cfr., ad esempio, il passo di Etica Nicomachea, V, 3, 1130 a 11-14 già ricordato «in che cosa, poi, differiscano la virtù e questo tipo di giustizia è chiaro da ciò che abbiamo detto: infatti, da un lato sono la stessa $\cos a$ ( $\dot{\eta} \alpha$ $\chi \dot{\tau})$, mentre dall'altro la 
avanzare solo la possibilità che la dimensione dell'ingiustizia si dia come sganciata da quella dell'ingiustizia? In che senso e a quali condizioni si può essere ingiusti senza essere viziosi? E, più in generale: è possibile che Aristotele non si sia reso conto di aver inficiato la sua riflessione etica, in uno snodo per di più assolutamente cruciale, attraverso una contraddizione così lampante? Oppure c'è qualche elemento che permetta di leggere (e di ri-leggere) il discorso aristotelico con una nuova chiave ermeneutica e di risolvere in questo modo la contraddizione?

L'elemento discriminante, come si diceva, è la presenza o meno della deliberazione, del proponimento, ovvero, in termini giuridici, della premeditazione. Ora, restando in ambito giuridico, l'elemento della premeditazione può costituire, in Aristotele ${ }^{63}$ come nel diritto contemporaneo, un'aggravante dell'azione ingiusta ( «l'azione ingiusta più grave... è quella più brutale; e poi quella maggiormente premeditata», leggiamo nella Retorica $)^{64}$ e il fondamento di una importante distinzione assiologica ${ }^{65}$ : la presenza o meno della premeditazione può infatti dar luogo ad un'azione più ingiusta

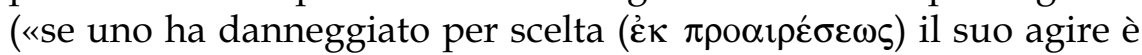
ingiusto e chi agisce ingiustamente in questo modo è propriamente

loro essenza non è la stessa ma, in quanto si dirige verso il prossimo è giustizia, in quanto considerata, di per sé, come un determinato stato abituale è virtù (corsivo mio)».

63 "Definiamo "commettere ingiustizia" come il danneggiare volontariamente e contrariamente alla legge... Si compie un'azione volontariamente quando si è consapevoli e non si viene forzati. Non tutte le azioni che si compiono volontariamente sono compiute in base a un proponimento, mentre tutte le azioni compiute in base a un proponimento sono compiute volontariamente: infatti, nessuno ignora ciò che si propone» (Retorica, I, 10, 1368 b 7-13).

${ }^{64}$ Retorica, I, 14, 1375 a 6-7.

${ }^{65} \mathrm{Si} \mathrm{cfr} .$, ad esempio, la nota distinzione (art. 43 cod. pen. "elemento psicologico del reato") tra "delitto doloso o secondo l'intenzione" (definito come delitto "preveduto e voluto come conseguenza della propria azione ed omissione»), "preterintenzionale o oltre l'intenzione" («quando l'azione od omissione deriva da un evento dannoso o pericoloso più grave di quello voluto dall'agente»), "colposo o contro l'intenzione" («quando l'evento... non è voluto dall'agente e si verifica a causa di negligenza o imprudenza o imperizia»).

Educ. e Filos., Uberlândia, v. 21, n. 41, p. 169-211, jan./jun. 2007. 


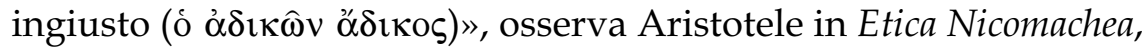
$\mathrm{V}, 10,1136$ a 1-2) e maggiormente punibile rispetto ad un'azione non premeditata. Fermo restando che, giuridicamente parlando, anche se compiuta in assenza di una deliberazione preventiva ${ }^{66}$, anche quando capita, come si racconta nella Retorica ${ }^{67}$, che uno che porta un anello ad una mano, sollevandola colpisca qualcuno (costui, infatti, «di fronte alla legge scritta» è comunque «colpevole e commette un reato ${ }^{68}$ ), l'azione è e rimane ingiusta, e come tale va punita dalla legge. Sia che l'atto ingiusto sia stato compiuto volontariamente o involontariamente, consapevolmente o inconsapevolmente, per scelta o meno, in ogni caso la legge è chiamata a pronunciarsi e ad emettere la sua sentenza. In questo senso si capisce perché, a fronte e in perfetta specularità rispetto alla "casistica etica" delle azioni ingiuste fornita nell'Etica Nicomachea ${ }^{69}$ e precedentemente ricordata, Aristotele, nella Retorica, riproponga la medesima classificazione in chiave giuridica:

tutte le imputazioni sono in rapporto o alla comunità o a un privato, e sono dirette contro chi ha agito o inconsapevolmente e involontariamente, o consapevolmente

\footnotetext{
${ }^{66}$ «Senza deliberazione preventiva, si ha sì un'azione ingiusta, come nel caso delle azioni che si compiono in preda all'impeto e di altre passioni» (Etica Nicomachea, V, 10, 1135 b 20-21). L. Grenet, Le droit pénal de la Grèce ancienne, in: AA.VV., Du châtiment dans la cité. Supplices corporels et peine de mort dans le monde antique, Table ronde organisée par l'École française de Rome avec le concours du Centre national de la recherche scientifique, Collection de l' École française de Rome, Rome 1984, p. 26, sottolinea come il dibattito filosofico sul tema della responsabilità, soprattutto in ambito penale, ha costituito il fulcro del pensiero filosofico e sofistico del V e del IV secolo: «le droit pénal occupe une large part dans la Politique des philosophes: les Lois de Platon transposent et intègrent toute la réflexion antérieure et voisent à l'accorder dans une synthèse avec les notices traditionelles; la théorie du delit chez Aristote... met l'accent moins sur le délit lui-même que sur son object et sur son suject: sur la victime créancière de la peine, individuelle ou collective».

${ }^{67}$ Retorica, I, 13, 1374 a 34 ss.

${ }^{68}$ Retorica, I, 13, 1374 a 35-1374 b 1.

${ }^{69}$ Cfr. Etica Nicomachea, V, 10, 1135 a 9 ss.
} 
e volontariamente, e, in questo caso, o con predeterminazione o spinto dall' emozione ${ }^{70}$.

D'altra parte la punizione dovrà certamente tener conto di tutti gli elementi in questione, come ad esempio il fatto che, a scatenare l'azione di chi agisce in preda all'impeto e quindi, secondo Aristotele, certamente non in modo premeditato, non è il soggetto che commette l'ingiustizia quanto colui che, scatenandone l'ira, ha messo in moto tutto il processo.

Per questo motivo, giustamente, si ritiene che le azioni che derivano dall'impeto non siano premeditate, dal momento che a innescare l'azione non è chi agisce in preda all'impeto ma chi lo ha fatto arrabbiare ${ }^{71}$.

\section{E ancora:}

Inoltre l'azione ingiusta è volontaria e dipende da una scelta e anteriore ad ogni provocazione (infatti chi per primo ha subito e per questo contraccambia, non si pensa che agisca ingiustamente $)^{72}$ (corsivo mio).

Un'osservazione interessante, soprattutto in quanto, nello spostamento, seppur parziale, dell'imputabilità dell' azione ingiusta dal soggetto agente ad un soggetto esterno, ritenuto responsabile dell'innesco di tutta la catena delle azioni, viene a delinearsi uno scenario che entra in aperto conflitto con la dimensione etica. In questo campo, infatti, l'azione dettata dall'impeto, al pari di quella mossa dal desiderio, qualora non venga subordinata ad una regola e non si conformi ad essa, costituisce sempre e comunque un errore $^{73}$. Il fatto che a scatenare l'ira sia stato un altro individuo

${ }^{70}$ Retorica, I, 13, 1373 b 34-37.

${ }^{71}$ Etica Nicomachea, V, 10, 1135 b 26-27.

${ }^{72}$ Etica Nicomachea, V , 15, 1138 a 20-22.

${ }^{73}$ Cfr. Etica Nicomachea, III, 3.

Educ. e Filos., Uberlândia, v. 21, n. 41, p. 169-211, jan./jun. 2007. 
non conta: in ogni caso, dal punto di vista etico, l'incapacità di esercitare un controllo sui propri impeti e sulle proprie passioni deve essere ritenuto irrimediabilmente colpevole.

\section{b) Azioni ingiuste e passioni: piano etico e piano giuridico a confronto}

Sulla questione della responsabilità ${ }^{74}$ delle proprie azioni e sul ruolo che le passioni (ira e desiderio in primis) giocano nell'attribuzione della responsabilità stessa, infatti, la posizione aristotelica è chiarissima. Mentre chi agisce per costrizione e per ignoranza può essere moralmente assolto, chi commette ingiustizia sotto la spinta delle passioni, agisce comunque volontariamente e deve essere ritenuto responsabile dei suoi $\mathrm{atti}^{75}$. Se infatti è vero, come ha osservato Vegetti ${ }^{76}$, che "Aristotele avrebbe recisamente negato che la passione (thymos ed epithymia) possa venire considerata fattore di involontarietà, a differenza della costrizione violenta e dell'ignoranza» e che quindi, in base a questo fondamentale assunto, «di fronte al tribunale della sua etica, Elena... non sarebbe uscita assolta» ${ }^{77}$, allora ci troviamo di fronte

${ }^{74}$ Per un'accurata indagine del tema della responsabilità nella teoria aristotelica dell' azione cfr. C. Natali, Responsabilità e determinismo nell'etica aristotelica, in AA.VV, Il dibattito etico e politico..., cit. pp. 481-509, e, sempre dello stesso autore, Aristotele: azione e responsabilità, in AA.VV. La libertà del bene, C. Vigna (a cura di), Vita e Pensiero, Milano 1998, pp. 85-103.

75 «Infatti, di certo, non dice bene chi afferma che gli atti compiuti a causa dell'impeto e del desiderio sono involontari. In primo luogo, infatti, più nessuno degli altri animali agirebbe volontariamente e nemmeno i bambini; e poi, che forse non compiamo volontariamente nessuna delle azioni causate da impeto e da desiderio, oppure compiamo volontariamente quelle moralmente belle, mentre involontariamente quelle turpi?» (Etica Nicomachea, III, 3, 1111 a 24-28). Anche in questo caso sembra interessante notare come la posizione aristotelica trovi un perfetto corrispettivo nelle vigenti norme di diritto penale. L' art. 90 del codice penale ("Stati emotivi o passionali") recita: "Gli stati emotivi o passionali non escludono né diminuiscono l'imputabilità».

${ }^{76}$ M. Vegetti, L'etica degli antichi, Laterza, Roma-Bari 1996, p. 102.

${ }^{77}$ Vegetti, Etica..., p. 102. In questo senso Aristotele si pone in una posizione antitetica rispetto a Gorgia che, nel suo Encomio di Elena, cerca appunto di 
ad un quadro radicalmente diverso rispetto al contesto giuridico abbozzato precedentemente.

Prendiamo l'esempio dell'adulterio, a cui lo stesso Aristotele fa spesso ricorso all'interno della propria indagine. Un adulterio, afferma Aristotele, al pari di un omicidio e di furto, è un'azione assolutamente riprovevole e sempre da condannare dal punto di vista morale. Si legge, infatti, in Etica Nicomachea, II, 6, 1107 a 1117 che

tutte queste cose e quelle simili sono biasimate per il fatto di essere vergognose in se stesse e non i loro eccessi nè i loro difetti. Riguardo ad esse, dunque, non è mai possibile comportarsi correttamente, ma si sbaglia sempre; infatti in casi come questi il bene e il suo opposto non consistono, ad esempio, nel commettere adulterio con la persona appropriata, quando e come si deve, ma in assoluto, il compiere una qualsiasi di quelle cose è sbagliato in assoluto ${ }^{78}$.

discolpare Elena dall'accusa di essere fuggita da Troia: quand'anche fosse stata spinta dalla passione erotica, infatti, essa andrebbe assolta, dal momento che la passione erotica costituisce, al pari della violenza fisica, una forma di costrizione rispetto alla quale si è vittime e non responsabili (Cfr. DK B11 6 ss).

${ }^{78} \mathrm{Cfr}$. anche Etica Eudemia, II, 3, 1221 b 18-26: «Inoltre non bisogna ignorare il fatto che per alcune delle passioni sopra ricordate, non vale il "come" vengono provate, se con il "come" si intende l'essere affetto più del dovuto. Per esempio l'adultero non è tale perché ha relazioni con donne sposate in misura maggiore di quanto dovrebbe (infatti non è questo), ma questa è, di per sé, un'azione perversa. Infatti, nel medesimo nome, sono compresi insieme sia la passione sia l'essere di una certa qualità. Lo stesso vale anche per la violenza. Perciò accade anche che contestino, sostenendo di avere avuto relazioni, ma di non aver compiuto adulterio; infatti o non sapevano o erano costretti; e dicono sì di aver inferto delle percosse, ma di non essere stati violenti. E lo stesso anche per altri casi di questo genere»; Grande Etica, I, 8, 1186 a 37-1186 b 3: «nel caso dell'adulterio e dell'adultero. Quest'ultimo, infatti, non è colui che rovina le donne libere "più del dovuto". Ma questo caso, così come altri di questo tipo che siano compresi nel piacere secondo incontinenza, è biasimevole in se stesso, a prescindere dalle nozioni di eccesso e di difetto».

Educ. e Filos., Uberlândia, v. 21, n. 41, p. 169-211, jan./jun. 2007. 
Una condanna senza appello ${ }^{79}$, quella che Aristotele emette contro chi si macchia della colpa di adulterio. Lasciandosi trascinare all'impeto della passione, accecato da un desiderio ${ }^{80}$ che lo spinge a compiere azioni dissennate o a mettere addirittura a repentaglio la propria esistenza, ridotto dalle proprie pulsioni a vivere come le bestie, o come gli schiavi ${ }^{81}$, l'adultero costituisce l'exemplum in negativo per eccellenza di un essere umano non all'altezza del proprio compito e del proprio nome.

Diverso, e decisamente meno monolitico, è il giudizio emesso dal tribunale della legge che, annoverando l'adulterio tra le azioni involontarie (dove per volontarie si deve intendere, come ha osservato Natali, "non volute da entrambi i soggetti in questione" ${ }^{82}$ ) e istituite di nascosto ${ }^{83}$, si pronuncia con toni meno duri nei confronti dell'adulterio. A patto, però che si tratti di un adulterio "autentico", compiuto cioè senza secondi fini, attuato sull'onda del desiderio e, quindi, anche senza premeditazione. Infatti, tra chi «commette

${ }^{79}$ Adkins, Merit..., p. 181 introduce, a questo proposito, una interessante precisazione che mostra come il giudizio etico sull'adulterio, all'interno della cultura greca, subisse delle oscillazioni a seconda che il soggetto adultero fosse l'uomo o la donna: mentre l'adulterio viene vissuto sempre come una vergogna per il marito offeso, al contrario, in casi normali (cioè a meno che, ad esempio, non si commetta adulterio con la moglie del proprio ospite) l'atto non è considerato vergognoso per colui che lo commette.

${ }^{80}$ "Infatti gli adulteri compiono molte azioni ardite spinti dal desiderio», si ricorda in Etica Nicomachea, III, 11, 117 a 1-2.

${ }^{81}$ Cfr. Etica Nicomachea I, 3.

82 «In questi casi il perpetratore agisce volontariamente, e la vittima subisce involontariamente, ma Aristotele chiama tali relazioni involontarie, perché considera, a quanto pare, volontarie solo le relazioni sociali in cui entrambe le parti in causa entrano per loro scelta» (Natali, Etica Nicomachea..., p. 490, n. 432).

83 «Dato che alcune relazioni sociali sono volontarie e altre involontarie; volontarie sono quelle come vendita, compera, prestito a interesse, garanzia, comodato, pegno, salario (sono dette volontarie perché l'origine di tali rapporti sociali è volontaria), mentre, di quelli involontari, alcuni si istituiscono di nascosto, come furto, adulterio, avvelenamento, prostituzione, corruzione di schiavi, omicidio doloso, falsa testimonianza, mentre altri sono violenti, come maltrattamenti, sequestro, omicidio, rapina, mutilazione, insulti, oltraggio» (Etica Nicomachea, V, 5, 1131 a 2-9).

Educ. e Filos., Uberlândia, v. 21, n. 41, p. 169-211, jan./jun. 2007 
adulterio per guadagnarci qualcosa e ne trae profitto» e chi «lo fa per desiderio, rimettendoci di tasca propria e incorrendo nella pena ${ }^{84} c^{\prime}$ è una differenza notevole dal punto di vista legale. Una differenza che si allarga enormemente quando si tratta di esaminare due diverse figure di adulterio o, più in generale, due diverse tipologie di azioni ingiuste: quelle compiute una sola volta e quelle compiute abitualmente. Si tratta, come è evidente, di una discriminante fondamentale dal punto di vista giuridico che ha anche delle importanti ricadute dal punto di vista più strettamente etico. Infatti, leggiamo in Etica Nicomachea, V, 10, 1134 a 19-22,

può accadere che uno abbia una relazione con una donna sapendo di chi si tratti, ma che lo faccia non a causa di una scelta razionale, ma perché spintovi dalla passione. Ora, egli compie certamente un'azione ingiusta ma non è ingiusto, come non è ladro anche se una volta ${ }^{85}$ ha rubato, né adultero anche se commise un adulterio; lo stesso vale per tutti gli altri casi.

Un passo di capitale importanza perché, oltre a riproporre l'alternativa, già accennata ma su cui si dovrà tornare tra poco, tra scelta razionale $v s$. passione, ci introduce a uno snodo cruciale per l'intera riflessione aristotelica della giustizia e dell'ingiustizia, che è quello dell'habitus ${ }^{86}$.

${ }^{84}$ Cfr. Etica Nicomachea, V, 4, 1130 a 25-26.

${ }^{85}$ L'aggiunta di questa specificazione è, a mio avviso, supportata dalla presenza

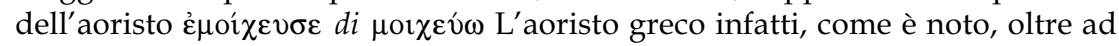
indicare un'azione puntuale nel passato, può assumere o un valore gnomico o, e questo è il caso, un valore ingressivo, «ad indicare l'incominciamento di un'azione o di uno stato» (A. Sivieri- P. Vivian, Corso di lingua greca, Casa Editrice G. D’Anna, Messina-Firenze 1986, p. 143).

${ }^{86} \mathrm{La}$ questione del nesso tra abitudine e legge in Aristotele e, più in generale, nella cultura e nella società greche, viene posta da J. De Romilly, La loi dans la pensée grecque des origines à Aristote, Les Belles Lettres, Paris 2001; trad. it. E. Lana: La legge nel pensiero greco. Dalle origini ad Aristotele, Garzanti, Milano 2005, in particolare alle pp. 201 ss. Uno studio ricco e documentato sul tema del

Educ. e Filos., Uberlândia, v. 21, n. 41, p. 169-211, jan./jun. 2007. 
Ma tornando per un attimo all'esempio dell'adultero ne risulta che, mentre dal punto di vista etico, la condanna è totale e definitiva, dal punto di vista giuridico il giudizio, emesso sulla base di una serie di valutazioni sempre secundum quid, risulta decisamente più indulgente e meno univoco.

Infatti, se l'azione è e resta comunque ingiusta e quindi da punire, nella formulazione della condanna bisogna tener conto per lo meno di due fondamentali attenuanti:

1) se l'adultero non ha secondi fini ma è mosso unicamente dal desiderio, la sua azione non può essere considerata premeditata;

2) se l'adultero ha commesso adulterio una sola volta non può essere considerato adultero incallito, nel senso che non ha stabilito una relazione adulterina che, come tale, interferisca con la vita coniugale.

Oltre all'esempio dell'adultero, poi, Aristotele costella periodicamente le sue riflessioni etiche di altri casi analoghi. Uno tra questi, riportato sia nell' Etica Nicomachea (VII, 7) che nella Grande Etica (II, 6) e, probabilmente, noto caso giudiziario dell'epoca, è quello dell' individuo incontinente che picchia il proprio padre. Ora, mentre dal punto di vista morale la sua condotta deve essere certamente condannata, perfino nel caso in cui riesca a controllare questo suo desiderio ${ }^{87}$ («non è continente colui che non ritiene vergognoso picchiare il proprio padre e, pur desiderando farlo, se ne astiene $\left.{ }^{88}\right)$, accade che il medesimo individuo, giuridicamente

rapporto tra legge e natura, soprattutto nelle sue implicazioni etiche e politiche,

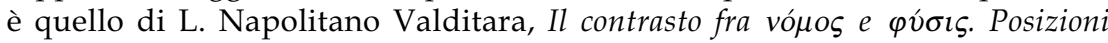
diverse e diverse indicazioni di condotta, in AA.VV. AA.VV, Il dibattito etico e politico in Grecia tra il V e il IV secolo, M. Migliori (a cura di), La Città del Sole, Napoli 2000, pp. 11-42.

${ }^{87}$ Sul tema della continenza e dell'incontinenza e sul loro complesso nesso con le nozioni di virtù e di vizio in Aristotele, mi permetto di rinviare al mio

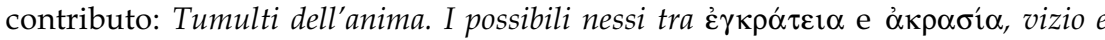
virtù nelle Etiche di Aristotele, in: Atti del Convegno: Attività e virtù. Anima e corpo in Aristotele. Macerata dal 24 al 26 marzo 2004 (in corso di pubblicazione).

${ }^{88}$ Grande Etica, II, 6, 1202 a 14-16. 
parlando, sia da considerare innocente: i giudici, ricorda Aristotele, accettarono la sua argomentazione («ma anche lui -disse- ha picchiato il suo e quello, a sua volta, aveva picchiato il proprio»e poi, mostrando il figlio: «e questo picchierà me -disse- quando sarà diventato uomo» ${ }^{89}$ ) e lo assolsero perché ritennero che si trattasse di una colpa o "tara congenita"

Ancora una volta, quindi, ci troviamo di fronte a un fenomeno che Aristotele permette di osservare da una duplice prospettiva: quella etica, da un lato, e quella giuridica, dall'altro, delineando così due diversi e irriducibili scenari in cui collocare e attraverso cui leggere e ricostruire la medesima "scena del misfatto".

\section{4) Giustizia e ingiustizia - azioni giuste e azioni ingiuste: la questione dell'habitus}

Il passo precedentemente preso in esame aveva messo in campo la questione dell'habitus, questione, come è noto, centrale per la riflessione etica dello Stagirita, e, insieme, snodo assolutamente cruciale per la trattazione del tema della giustizia e dell'ingiustizia.

Gli uomini credono che il commettere ingiustizia dipenda da loro e, per questo, ritengono che l'essere giusti sia facile. Ma in realtà non è affatto così. Infatti, per certi versi, è facile e dipende da loro avere una relazione con la moglie del vicino, picchiare il prossimo, corrompere col denaro, ma fare ciò sulla base di uno stato abituale acquisito non è facile né dipende da loro,

si legge nella Nicomachea ${ }^{91}$.

\footnotetext{
${ }^{89}$ Etica Nicomachea, VII, 7, 1149 b 9-11.

${ }^{90}$ In Grande Etica, II, 6, 1202 a 23-26 Aristotele riporta «la storia di quel figlio che una volta, condotto in tribunale perché picchiava il padre, si difese dicendo "anche costui picchiava il proprio padre" e così fu assolto; infatti ai giudici sembrò che la colpa fosse congenita».

${ }^{91}$ Etica Nicomachea, V, 13, 1137 a 5-9.
}

Educ. e Filos., Uberlândia, v. 21, n. 41, p. 169-211, jan./jun. 2007. 
L'agire giustamente (o ingiustamente) viene, dunque, svincolato dalla giustizia (e dall'ingiustizia): una cosa è commettere un'azione, giusta o ingiusta che sia, un'altra è essere giusti o ingiusti, cioè possedere la giustizia (o l'ingiustizia) come habitus, cioè come stato abituale. Ed è esattamente in questi termini che la giustizia e l'ingiustizia vengono definite all'inizio del V libro della Nicomachea:

vediamo... che tutti intendono con "giustizia" quello stato abituale da cui gli uomini sono resi in grado di compiere azioni giuste e sulla base del quale agiscono giustamente e vogliono le cose giuste; lo stesso vale anche per l'ingiustizia, sulla base della quale compiono azioni ingiuste e vogliono cose ingiuste.

Quindi, la giustizia e l'ingiustizia, nel loro configurarsi come stati abituali radicati nell'individuo, costituiscono le condizioni di possibilità della realizzazione di azioni giuste e ingiuste. In questo senso giustizia e azioni giuste da un lato, ingiustizia e azioni ingiuste dall'altro, si danno come ambiti strettamente legati e interdipendenti. Non si può agire giustamente, ci dice Aristotele, se non all'interno dell'alveo della giustizia, e cioè se non perché la giustizia, in quanto disposizione, ha orientato il soggetto in direzione sia del volere sia del compiere azioni giuste. E lo stesso discorso può essere fatto a proposito dell'ingiustizia.

D'altra parte, però, sia nei passi esaminati precedentemente, sia in un'icastica affermazione di Etica Nicomachea, V, 10, 1135 a $16-17$, il legame tra ingiustizia e azioni ingiuste viene nuovamente spezzato

È possibile compiere azioni ingiuste senza essere ancora

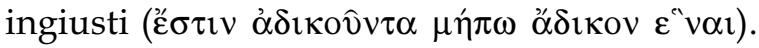

In realtà, quella che potrebbe a prima vista apparire come una contraddizione, risulta facilmente scioglibile se letta e inquadrata all'interno del sistema categoriale aristotelico.

L' $\xi^{\prime} \xi 1 \zeta$, infatti, come Aristotele spiega diffusamente all'interno delle sue riflessioni etiche implica un lungo e complesso processo 
di costruzione e di sedimentazione del proprio agire e del proprio sé. Infatti, si ricorda sempre nell' Etica Nicomachea ${ }^{92}$ «le virtù non si generano né per natura né contro natura, ma è nella nostra natura accoglierle, e sono portate a perfezione in noi per mezzo dell'abitudine... acquistiamo le virtù perché le abbiamo esercitate in precedenza, come avviene anche nel caso di tutte le arti. Quello che si deve fare quando si è appreso, facendolo, lo impariamo: per esempio, costruendo si diviene costruttori e suonando la cetra, citaristi; e allo stesso modo compiendo atti giusti si diventa giusti, temperanti con atti temperanti, coraggiosi con atti coraggiosi». Quindi: come la ripetizione continua di un'attività instaura l'abilità tecnica, così a forza di compiere azioni virtuose si acquisisce la virtù, intesa come disposizione, come habitus ${ }^{93}$. Un parallelismo che Aristotele trae dalla vita quotidiana ma che esprime a pieno la funzione plasmatrice dell'abitudine (secondo la nota massima $\pi \hat{\alpha} v$ $\hat{\eta} \theta \circ \varsigma \delta$ ì $\grave{c} \theta 0 \varsigma$ che troviamo espressa nelle Leggi di Platone ${ }^{94}$ ). Quello che, in un primo tempo, viene eseguito in modo meccanico e costrittivo (perché chi inizia a compiere atti virtuosi lo fa inizialmente controvoglia, così come il giovane costruttore di case, inizialmente, si sarà sottoposto con difficoltà e in modo imperfetto

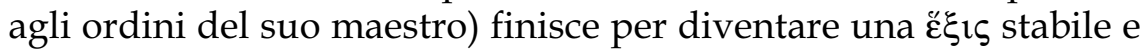

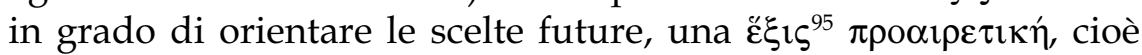

${ }^{92}$ Etica Nicomachea, II, 1, 1103 a 26-1103 b 2.

93 "Virtue for Aristotle is a state of character which manifests itself in choice or preference (hexis prohairetike)» (W. F. R. Hardie, Aristotle's ethical theory, Clarendon Press, Oxford 1968, 19992, p. 102).

${ }^{94}$ Platone, Leggi VII, 792 E 2: «Per effetto dell'abitudine... si imprime in noi in modo indelebile il carattere» (Platone, Leggi, trad. it. R. Radice in: Platone, Tutti gli scritti, a cura di G. Reale, Rusconi, Milano 1991).

95 «Per alcuni aspetti, il termine aristotelico hexis corrisponde al meglio al nostro termine "disposizione" piuttosto che ad "abito"; una virtù come il coraggio è una disposizione, perché è la condizione per la quale sono disposto in modo da agire coraggiosamente; $l^{\prime}$ hexis indica proprio questa condizione. Tuttavia, "abito", è la traduzione del termine hexis che si è ormai consolidata» (J. Annas, The Morality of Happiness, Oxford University Press, 1993; trad. it. M. Andolfo: La morale della felicità in Aristotele e nei filosofi dell'età ellenistica, prefazione di G. Reale, Vita e Pensiero, Milano 1998, p. 80).

Educ. e Filos., Uberlândia, v. 21, n. 41, p. 169-211, jan./jun. 2007. 
"disposizione implicante una scelta" o, meglio, una "disposizione che orienta la scelta". Il che significa, contemporaneamente, «che la virtù si costituisce a seguito di scelte ripetute e dallo sviluppo di abitudini di scelta» ${ }^{96}$. Detto in altri termini: una serie di scelte determina un abito e ogni abito determina delle scelte ${ }^{97}$, rendendo legittima l'affermazione secondo cui «una virtù (o un vizio) è il modo in cui mi sono "coltivato" e in cui ho scelto di essere»" ${ }^{98}$.

Ogni scelta, insomma, ha un passato e un futuro ed è il risultato di un determinato modo di agire, di un determinato modo di scegliere che conferisce uno stile al nostro comportamento e al nostro stesso modo di essere. «Ogni azione che compiamo riflette il modo in cui ci siamo comportati e influenza la maniera in cui ci comporteremo» ${ }^{99}$, ha affermato Julia Annas, esprimendo efficacemente quella tipica circolarità a "spirale" costituita dal rapporto tra virtù, scelta e azione umane. Assolutamente emblematico risulta, in questo senso, un passo di Etica Nicomachea, III, 7, 114 a 11-19 che merita di essere riportato per intero proprio per la sua straordinaria incisività:

Inoltre è assurdo che chi compia ingiustizie non voglia essere ingiusto... Non per questo, però, gli è possibile smettere di essere ingiusto e di diventare giusto quando vuole. Infatti neppure chi è malato può diventare sano, anche se si dà il caso che si sia ammalato volontariamente, vivendo in modo sfrenato e non dando ascolto ai medici: infatti, all'inizio, vi era per lui la possibilità di non ammalarsi, ma una volta che si è lasciato andare ciò non è più possibile, allo stesso modo in cui, per chi ha lanciato una pietra, non è più possibile riprenderla, anche se dipendeva da lui il prenderla e scagliarla, dal momento che il principio è in lui (corsivo mio).

\footnotetext{
${ }^{96}$ Annas, Morale..., p. 82.97 L'abito può essere definito, per dirla con P. Donini, Ethos. Aristotele e il determinismo, Edizioni dell'Orso, Alessandria 1989, p. 75, come «un principio di risposte costanti a situazioni di un certo tipo, omogenee fra loro e omogenee, inoltre, alle attività che portarono alla formazione dell'abito stesso».98 Annas, Morale..., p. 80.

${ }^{99}$ Annas, Morale..., p. 83.
} 
Il radicamento dell'habitus nell'individuo, la sua crescita "a valanga" e la ricaduta del comportamento su se stesso non potevano essere espressi da Aristotele in modo più efficace. La scelta iniziale, esemplificata dalla possibilità di lanciare o meno la pietra, costituisce il momento di avvio di un processo che, se certamente non è totalmente vincolato a priori, è pur sempre dotato di un significativo margine di irreversibilità: una volta assunto l'habitus dell'ingiustizia non si può smettere di essere ingiusti quando si vuole, esattamente come non si può pretendere di tornare immediatamente sani una volta che ci si è ammalati.

Ma se, in quanto $\varepsilon^{\prime} \xi \varepsilon 15$, la giustizia e l'ingiustizia si configurano, rispettivamente, come virtù e come vizio, dato che uno dei profili del vizio e della virtù è costituito proprio dallo stato abituale, permettendo una ricostituzione del problematico legame giustiziavirtù/ingiustizia-vizio, d'altro canto l'apertura a questa nuova dimensione della virtù ci permette di comprendere il senso di un possibile divorzio tra le due dimensioni. Affermare infatti, come si fa nel passo di Etica Nicomachea $\mathrm{V}$ già richiamato, che, tra le azioni ingiuste compiute volontariamente, solo quelle deliberate e quelle scelte possono essere viziose, significa far giocare la nozione di virtù e di vizi come habiti orientativi della condotta dell'essere umano $^{100}$. In altre parole: mentre chi si è comportato ingiustamente solo perché si è lasciato trascinare dalla passione, pur compiendo un'azione riprovevole e giuridicamente perseguibile, non è vizioso, chi ha deliberato e scelto di mettere in atto un'azione ingiusta è pienamente vizioso, dal momento che l'ingiustizia, stato abituale

100 «Per abitudine - si legge in Retorica, I, 10, 1369 b 5-6- [si verificano] quelle azioni che si compiono per il fatto di averle compiute spesso». In questo senso si può affermare, come ha fatto giustamente G. Romeyer Dherbey, La question du droit naturel (Éthique à Nicomaque V 10, 1134 b 19-1135 a 6), in AA. VV., L'excellence de la vie. Sur «l'Éthique à Nicomaque» et «l' Éthique à Eudème» d' Aristote, G. Romeyer Dherbey (dir.), G. Aubry (éd.), Vrin, Paris 2002, pp. 126-127, che «on peut donc commetre un adikêma sans être adikos. Pourquoi? Parce que cet acte n'a pas été fait par choix délibéré... Aristote fait donc intervenir ici le côté subjectif de l'acte, son aspect intentionnel et réfléchi».

Educ. e Filos., Uberlândia, v. 21, n. 41, p. 169-211, jan./jun. 2007. 
in lui già radicato in seguito ad altre scelte precedenti, ha nuovamente orientato la scelta in quella direzione e lo ha di nuovo condotto sulla via del vizio.

Nello stesso modo sia i giusti sia gli ingiusti, sia gli altri che

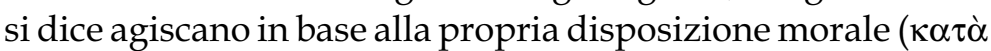
$\tau \grave{\alpha} \varsigma$ है $\xi \varepsilon 1 \varsigma)$, agiranno per questi motivi: o per ragionamento o per emozione. Ma alcuni agiranno in base a caratteri ed emozioni positivi, altri in base a quelli opposti

si legge nella Retorica ${ }^{101}$. La spirale virtuosa del giusto, cioè di colui che, in quanto giusto, è sempre più in grado di scegliere di compiere azioni giuste, di farlo sempre meglio e senza fatica, si trasforma, nel caso dell'ingiusto, in un circolo vizioso sempre più opprimente e vincolante, che finisce per incatenare sempre più fortemente e irrimediabilmente l'ingiusto al proprio stato abituale.

Inoltre l'elemento dell'habitus, che ha una valenza prettamente etica, ha una ricaduta anche sul terreno squisitamente giuridico. L'ingiusto incallito, infatti, cioè colui che viene indotto a compiere ripetutamente azioni ingiuste dal proprio stato abituale, è colui che, in termini giuridici, viene detto recidivo ${ }^{102}$. E la recidiva costituisce certamente un'aggravante della colpa stessa ${ }^{103}$ :

è più grave... rendersi spesso colpevole dello stesso errore

troviamo scritto nella Retorica ${ }^{104}$

D'altro canto il consiglio che lo Stagirita impartisce ai giudici, sempre nella Retorica, è proprio quello di tener conto della personalità complessiva di colui che commette il reato, piuttosto che limitarsi ad attenersi il singolo fatto: «è equo.... considerare

${ }^{101}$ Retorica, I, 10, 1369 15-18.

${ }^{102}$ Cfr. Codice Penale, capo II, “Della recidiva, dell'abitualità e professionalità nel reato e della tendenza a delinquere".

${ }^{103}$ Cfr. anche sopra, a proposito dell'esempio dell'adulterio.

${ }^{104}$ Retorica, I, 14, 1375 a 4-5. 
non il fatto in se stesso ma secondo l'intenzione; non la parte ma il tutto; non come è ora un uomo, ma come è stato sempre o nella maggioranza dei casi» ${ }^{105}$. La necessità di disporre di un quadro completo della personalità e della condotta dell'individuo per valutare la singola azione può giocare, come è ovvio, a sfavore o a favore dell'individuo, costituendo un importante "effetto alone" nella valutazione dell'evento criminoso: uno stato abituale corrotto o degenere influenzerà negativamente il parere dei giudici aggravando la posizione del reo, mentre un habitus retto rappresenterà certamente un'attenuante della colpa commessa.

La questione della giustizia e dell'ingiustizia come habiti permette, dunque, da un lato di comprendere che, per essere giusti o ingiusti, non basta compiere atti di giustizia e di ingiustizia ${ }^{106}$ (cioè che ci siano alcuni, come si ricordava, che «agiscono sì ingiustamente, e le loro azioni sono sì ingiuste, ma non per questo sono ancora ingiusti o viziosi» ${ }^{107}$, esattamente come è possibile agire coraggiosamente senza per questo essere coraggiosi, afferma altrove Aristotele ${ }^{108}$ ) e, dall'altro, di comprendere il senso di quella problematica scissione tra giustizia e azione giusta da un lato e ingiustizia e azione ingiusta dall'altro. Allora, come nell'esempio dell'adultero ricordato precedentemente, mentre è certamente facile compiere un'azione giusta, farlo sulla base di uno stato abituale acquisito non lo è affatto ${ }^{109}$, esattamente come c'è una sostanziale differenza tra chi agisce giustamente perché costrettovi dalla legge

${ }^{105}$ Retorica, I, 13, 1374 b 10-16.

106 «Aristote n'oublie jamais que la justice ne se réduit pas à faire des choses justes, qu'elle exige au contraire des dispositions intérieures» (Gauthier-Jolif, Éthique..., Tome II, Commentaire, p. 328).

${ }^{107}$ Etica Nicomachea, V, 10, 1135 b 22-24.

${ }^{108}$ Etica Nicomachea, III, 9, 1115 a 16 ss.

${ }^{109}$ Cfr. il passo, già ricordato, di Etica Nicomachea, V, 13, 1137 a 5-9. Come hanno osservato Gauthier-Jolif, Éthique..., Tome II, Commentaire, p. 420, «la justice est en notre pouvoir, certes, mais on n'y parvient pas sans exercice, car l'essentiel n'est pas de faire des choses justes, c'est de les faire de la bonne façon... La même chose va être affirme du vice... c'est que le vice est lui aussi une disposition intérieure».

Educ. e Filos., Uberlândia, v. 21, n. 41, p. 169-211, jan./jun. 2007. 
e chi, invece, lo fa sulla base della propria disposizione morale, «senza costrizione» ${ }^{110}$ e traendo piacere dal compiere atti giusti ${ }^{111}$. In questo senso lo $\sigma \pi 0 v \delta \alpha \hat{\imath} o \varsigma$ $\alpha v \eta ́ p$ aristotelico, cioè l'individuo giusto e moralmente retto, anche se fosse dotato dell'anello di Gige, di quel prodigioso anello che, rendendo invisibili, racconta Platone nella Repubblica ${ }^{112}$, assicura l'impunità dalle sanzioni (facendo sì che neppure chi è giusto riesca a tenersi «lontano dai beni altrui, e... non toccarli, quando invece avrebbe la possibilità di prendere impunemente dai banchi del mercato tutto quello che desidera, di penetrare nelle case e fare l'amore con chi gli aggrada, di uccidere e di liberare dai ceppi chi vuole $\left.\left.{ }^{113}\right\rangle\right)$, resterebbe integro proprio in virtù del suo stato abituale, manterrebbe la sua rettitudine proprio in virtù del suo solido habitus.

\section{Conclusioni}

Questa breve indagine attraverso le nozioni di giustizia e ingiustizia, virtù e vizio nello Stagirita, ci ha permesso di individuare alcune costanti del pensiero aristotelico e di sciogliere alcune apparenti contraddizioni del discorso stesso. Una costante di questo pensiero è costituita indubbiamente dal progressivo allargamento e dalla continua complessificazione degli schemi espositivi, di chiavi ermeneutiche, sempre duttilissime e pronte ad adattarsi all'estrema variabilità e polivocità del reale. Si tratta di un modo di procedere

${ }^{110}$ Retorica I, 1375 a 16-17. «l'uomo migliore è quello che risulta giusto senza costrizione». Sul tema dell'adesione forzata e spontanea alla legge cfr. G. F. Falchi, Le basi morali del diritto penale Zannoni Editore, Padova 1930, pp. 44-45: «coloro che dapprima si piegheranno nolentemente alla imposizione legale, dipoi vi si assoggetteranno per la razionalità della norma, per il consolidamento in loro della convinzione di quella osservanza, e quindi la norma sarà naturalmente adempita per la propensione spontanea».

${ }^{111}$ Infatti, come ricorda lo Stagirita in Etica Nicomachea, X, 2, 1173 b 29-30, «non è possibile godere del piacere della giustizia senza essere giusti».

${ }^{112}$ Cfr. Platone, Repubblica, II, 359 e ss.

${ }^{113}$ Platone, Repubblica, II, 360 b 5- c 3; trad. it. R. Radice, in: Platone, Tutti gli scritti (a cura di G. Reale), Rusconi, Milano 1991. 
che, se da un lato riesce pienamente nell'intento di rispettare la ricchezza e la polivalenza della materia da esaminare, di un terreno così variegato e sfuggente come quello dell' azione umana, dall'altra è destinato a destare inevitabilmente lo smarrimento del lettore che cerca faticosamente di porsi sulle orme dell'argomentazione del Filosofo.

In questo faticoso tentativo si è cercato di non ridurre l'estrema ampiezza e polivalenza delle nozioni di giustizia e di virtù in Aristotele e di non cadere in quella diffusa e pericolosa tentazione semplificazionistica propria di gran parte della tradizione esegetica ( «l'interpretazione tradizionale, che andava alla ricerca di distinzioni rigorose, ha finito per identificare tout court la giustizia... con la virtù totale, e a separare dalla prima la giustizia particolare» ${ }^{114}$ ).

Virtù come parte, virtù come tutto, virtù come giusto mezzo, virtù come disposizione che orienta la scelta, virtù come facoltà in grado di distribuire i beni: la giustizia, così come la riflessione aristotelica ci autorizza ad affermare, è tutto questo, e per essere compresa a pieno va colta in tutte le sue accezioni. Accezioni, per di più, mai pienamente esplicitate eppure sempre imprescindibilmente sottese al discorso dello Stagirita, che il Filosofo mette in campo facendole giocare a turno, in modo alterno, rendendo così ancora più disagevole il già l'arduo cammino interpretativo del lettore.

D'altra parte, è solo conoscendone e accettandone le regole che il gioco aristotelico può essere adeguatamente "giocato", senza cedere a facili tentazioni riduzionistiche e senza doversi arrendere alla constatazione di posizioni contraddittorie all'interno del testo stesso. Ed è solo facendo giocare tutti i sensi che la nozione di giustizia possiede che si può comprendere come, ad esempio, sia possibile affermare contemporaneamente che uno stesso atto ingiusto, da un certo punto di vista, è vizioso mentre, dall'altro, non lo è. L'ingiustizia, infatti, può essere considerata come vizio

${ }^{114}$ Giuliani, Definizione aristotelica..., p. 75.

Educ. e Filos., Uberlândia, v. 21, n. 41, p. 169-211, jan./jun. 2007. 
"totale", cioè come la totalità del vizio che unifica e dà senso alle sue parti, anch'esse viziose, tra cui risiede l'ingiustizia "parziale" stessa; può essere considerata come eccesso e difetto rispetto alla medietà virtuosa costituita dalla giustizia; può essere considerata come habitus, cioè come stato abituale che orienta le scelte. E l'atto ingiusto, dal canto suo, mentre per un verso non può non essere intimamente innervato dal vizio, dato che l'ingiustizia è vizio, ed è vizio come tutto e come parte (e dato anche che l'ingiustizia, in quanto eccesso e difetto nel distribuire e nell'attribuire i beni, è chiamata ad incarnare gli estremi viziosi rispetto al giusto mezzo virtuoso), per un altro verso non è vizio (o non lo è necessariamente), se per vizio si intende quella disposizione interiore, radicatasi nell'individuo in seguito ad una determinata condotta di vita, consolidata attraverso una serie di scelte e in grado di influenzare le scelte future. In altre parole Aristotele ci insegna che, anche se può sembrare strano, si può agire ingiustamente senza essere ingiusti, si possono commettere atroci delitti senza, per questo, essere viziosi, e non solo per la banale constatazione che si possono commettere ingiustizie per sbaglio, per imperizia o per ignoranza. Infatti di azioni ingiuste e, contemporaneamente, non viziose, si può macchiare chi si lascia andare all'ira, chi, pur in piena consapevolezza, si lascia travolgere dalla passione, chi, una volta, cede alla tentazione o al bisogno di commettere un furto. Azioni legalmente perseguibili, naturalmente, e anche azioni eticamente riprovevoli. Eppure non viziose. E non viziose perché, come abbiamo visto, c'è un'accezione del vizio (e, quindi, anche della virtù) che Aristotele chiama in causa ripetutamente all'interno della propria riflessione sulla giustizia e sull'ingiustizia, in base alla quale vizioso può essere detto solo colui ha uno stato abituale che lo caratterizza stabilmente (un delinquente abituale, diremmo in termini giuridici) e, sulla base di esso, delibera sulle proprie azioni e sceglie quelle da compiere. E lo stesso vale per la giustizia e per ogni atto giusto, il cui nesso rispetto alla nozione di virtù va stabilito volta per volta, in base al punto di vista assunto, a seconda della prospettiva di partenza. Come è stato giustamente osservato, «la dottrina aristotelica della giustizia può quindi essere studiata 
attraverso una serie di categorie che, mentre ne dimostrano la fondamentale articolazione su distinti livelli, complicano non poco questo settore della riflessione aristotelica» ${ }^{115}$.

Tò ôv $\pi \mathrm{o} \lambda \lambda \alpha \chi \hat{\omega} \varsigma \mathrm{c} \varepsilon \dot{\gamma} \gamma \tau \alpha \alpha \mathrm{l}:$ "l'essere si dice in molti modi" recita la celebre affermazione aristotelica. Un'affermazione che va presa sul serio e va intesa in senso tecnico: l'essere si declina sempre al plurale, la realtà è sempre molteplice e, per essere afferrata, ha bisogno di un linguaggio e di un pensiero a maglie larghe, che lascino vivere la sua debordante molteplicità senza soffocarla. C'è un bellissimo passo di Metafisica, K, 9 (1066 a 20-26) che merita di essere ricordato proprio per la sua capacità di esprimere questo concetto in modo esemplare:

Il movimento è evidentemente un atto, ma è un atto incompleto: ed appunto per questo è difficile comprendere che cosa sia il movimento. Ridurlo a privazione o a potenza o ad atto puro non è possibile: pertanto non resta che la spiegazione che noi abbiamo dato: il movimento è atto e non è atto, e questo è difficile da comprendere, ma è possibile (corsivo mio).

Solo attraverso il paziente attraversamento delle svariate accezioni di giustizia e di ingiustizia, di azione giusta e di azione ingiusta, incomprensibili a prescindere dal sottile e complesso legame con i molteplici sensi di virtù e di vizio, e destinati ad incrociarsi con questioni di notevole rilevanza etica e giuridica come la responsabilità morale del soggetto agente e le dinamiche psicologiche sottostanti alle azioni, sembra possibile illuminare, almeno in parte, lo sterminato affresco dell'agire umano realizzato da Aristotele.

${ }^{115}$ Zanetti, Nozione di giustizia..., p. 117.

Educ. e Filos., Uberlândia, v. 21, n. 41, p. 169-211, jan./jun. 2007. 


\section{Bibliografia citata e utilizzata}

\section{Testi antichi}

Aristotele, Etica Eudemia, trad. mia.

Aristotele, Etica Nicomachea, trad. mia.

Aristotele, Etica Nicomachea, traduzione, introduzione e note di C. Natali, Laterza, Roma-Bari 1999.

Aristotele, Grande Etica, trad. mia.

Aristotele, Metafisica, introduzione, traduzione, note e apparati di G. Reale, Appendice bibliografica di R. Radice, Bompiani, Milano 2000.

Aristotele, Retorica, Introduzione di F. Montanari, Testo critico, traduzione e note a cura di M. Dorati, Mondadori, Milano 1996.

Aristotele, Topici, introduzione, traduzione e commento di A. Zadro, Loffredo, Napoli 1974.

Platone, Leggi, trad. it. R. Radice in: Platone, Tutti gli scritti, (a cura di G. Reale), Rusconi, Milano 1991.

Platone, Repubblica, trad. it. R. Radice, in: Platone, Tutti gli scritti..., cit.

\section{Dizionari e strumenti}

Paliero C. E., Codice Penale e Normativa Complementare, 2001-2002, quinta edizione, Raffaello Cortina Editore, Milano 2001.

Rocci L., Vocabolario greco-italiano, Società Editrice Dante Alighieri, Città di Castello 1985. 
Sivieri A. - Vivian P., Corso di lingua greca, Casa Editrice G. D'Anna, Messina-Firenze 1986.

\section{Letteratura secondaria}

AA.VV. Aristotle Critical Assessments, Lloyd P. Gerson (ed.), 4 voll., Routledge, London-New York 1999.

AA.VV. Gigantomachia. Convergenze e divergenze tra Platone e Aristotele, M. Migliori (a cura di) Morcelliana, Brescia 2002.

AA.VV., Du châtiment dans la cité. Supplices corporels et peine de mort dans le monde antique, Table ronde organisée par l'École française de Rome avec le concours du Centre national de la recherche scientifique, Collection de l' École française de Rome, Rome 1984.

AA. VV., L'excellence de la vie. Sur «l'Éthique à Nicomaque» et «l' Éthique à Eudème»d' Aristote, G. Romeyer Dherbey (dir.), G. Aubry (éd.), Vrin, Paris 2002.

AA.VV, Il dibattito etico e politico in Grecia tra il $V$ e il IV secolo, M. Migliori (a cura di), La Città del Sole, Napoli 2000.

AA.VV. La libertà del bene, C. Vigna (a cura di), Vita e Pensiero, Milano 1998.

Adkins. A. W. H., Merit and Responsibility. A Study in Greek Values, Clarendon Press, Oxford 1960.

Annas J., The Morality of Happiness, Oxford University Press, 1993; trad. it. M. Andolfo: La morale della felicità in Aristotele e nei filosofi dell'età ellenistica, prefazione di G. Reale, Vita e Pensiero, Milano 1998.

Educ. e Filos., Uberlândia, v. 21, n. 41, p. 169-211, jan./jun. 2007. 
Berti E., Il filosofo e la città secondo Aristotele, in: Tatasciore C. (a cura di), I filosofi e la città..., cit., pp. 37-50.

Bretone M., Talamanca M., Il diritto in Grecia e a Roma, Laterza, Roma-Bari 1981.

Curzer H. J., Aristotle's Account of the Virtue of Justice, "Apeiron", XXVIII, 3 (1995), pp. 207-238.

D'Avenia M., L'aporia del bene apparente. Le dimensioni cognitive delle virtù morali in Aristotele, Vita e Pensiero, Milano 1998.

De Romilly J., La loi dans la pensée grecque des origines à Aristote, Les Belles Lettres, Paris 2001; trad. it. E. Lana: La legge nel pensiero greco. Dalle origini ad Aristotele, Garzanti, Milano 2005.

Donini P., Ethos. Aristotele e il determinismo, Edizioni dell'Orso, Alessandria 1989.

Falchi G. F., Le basi morali del diritto penale, Zannoni Editore, Padova 1930.

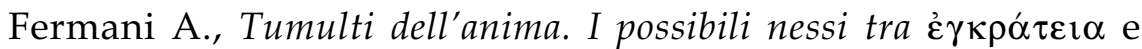

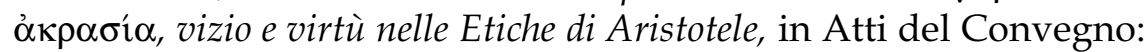
Attività e virtù. Anima e corpo in Aristotele. Macerata, 24-26 marzo 2004 (in corso di pubblicazione).

Gauthier R. A. - Jolif J. Y., L'Éthique à Nicomaque, deuxième édition avec une introduction nouvelle, 4 voll., Éditions Peeters, Louvainla Neuve, Paris 2002.

Giuliani A., La definizione aristotelica della giustizia. Metodo dialettico e analisi del linguaggio normativo, C. L. E. U. P., Perugia 1971.

Grenet L., Le droit pénal de la Grèce ancienne, in: AA.VV., Du châtiment dans la cité..., cit., pp. 9-35. 
Hardie W. F. R., Aristotle's ethical theory, Clarendon Press, Oxford $1968,1999^{2}$.

Kraut R., Are there Natural Rights in Aristotle?, "The Review of Metaphysics» 49 (1996), pp. 755-774, ora in: Lloyd P. Gerson (ed.), Aristotle Critical Assessments, 4 voll., vol. IV "Politics, Rhetoric and Aesthetics"..., cit. pp. 31-47.

Leyden W. von, Aristotle on Equality and Justice. His Political Argument, Macmillan, London 1985.

Migliori M., Misure ed etica tra quantità e qualità, «Tutto misure», 3, 1999, pp. 231-239 (I parte); «Tutto misure», 1, 2000, pp. 28-32 (II parte).

Miller, F. D. Jr., Aristotle and the Origins of Natural Rights, "The Review of Metaphysics» 49 (1996), pp. 873-907, ora in Lloyd P. Gerson (ed.), Aristotle Critical Assessments, 4 voll., vol. IV "Politics, Rhetoric and Aesthetics"..., cit., pp. 1-30.

Miller F. D. Jr., Nature, Justice and Rights in Aristotle's Politics, Clarendon Press, Oxford 1995.

Napolitano Valditara L., Il contrasto fra vó $\mu$ s e $\varphi v ́ \sigma ı \varsigma$. Posizioni diverse e diverse indicazioni di condotta, in AA.VV. Il dibattito etico e politico..., cit., pp. 11-42.

Natali C., Aristotele: azione e responsabilità, in AA.VV. La libertà del bene..., cit., pp. 85-103.

Natali C., Responsabilità e determinismo nell'etica aristotelica, in AA.VV, Il dibattito etico e politico..., cit., pp. 481-509.

Quitidamo N., Il concetto filosofico di "giustizia" in Aristotele e la sua inerenza col diritto, Stabilimento Tipografico Editoriale, Napoli 1935.

Educ. e Filos., Uberlândia, v. 21, n. 41, p. 169-211, jan./jun. 2007. 
Romeyer Dherbey G., La question du droit naturel (Éthique à Nicomaque V 10, 1134 b 19-1135 a 6), in AA. VV., L'excellence de la vie..., cit., pp. 125-137.

Rossetti L., Non solo dottrine politiche. L'apporto di Platone, Aristotele, Teofrasto e altri peripatetici alla cultura giuridica, in: M. Migliori (ed.), Gigantomachia..., cit., pp. 357-377.

Santas G., Goodness and Justice. Plato, Aristotle, and the Moderns, Blackwell, Oxford 2001.

Stern-Gillet S., Aristotles Philosophy of Friendship, State University of New York Press 1995.

Tatasciore C. (a cura di), I filosofi e la città, La Città del Sole, Napoli 2003.

Vegetti M., L'etica degli antichi, Laterza, Roma-Bari 1996.

Zanetti. G, La nozione di giustizia in Aristotele. Un percorso interpretativo, Il Mulino, Bologna 1993.

Data de registro 09/06/06

Data de aceite 10/07/06 